\title{
Testing the Sorption Hypothesis in Olfaction: A Limited Role for Sniff Strength in Shaping Primary Odor Representations During Behavior
}

\author{
Tristan Cenier, ${ }^{1,2}$ John P. McGann, ${ }^{1}$ Yusuke Tsuno, ${ }^{1}$ Justus V. Verhagen, ${ }^{1}$ and Matt Wachowiak ${ }^{1,2}$ \\ ${ }^{1}$ Department of Biology, Boston University, Boston, Massachusetts 02215, and ${ }^{2}$ Brain Institute, University of Utah, Salt Lake City, Utah 84112
}

The acquisition of sensory information during behavior shapes the neural representation, central processing, and perception of external stimuli. In mammals, a sniff represents the basic unit of odor sampling, yet how sniffing shapes odor representations remains poorly understood. Perhaps the earliest hypothesis of the role of sniffing in olfaction arises from the fact that odorants with different physicochemical properties exhibit different patterns of deposition across the olfactory epithelium, and that these patterns are differentially affected by flow rate. However, whether sniff flow rates shape odor representations during natural sniffing remains untested, and whether animals make use of odorant sorption-airflow relationships as part of an active odor-sampling strategy remains unclear. We tested these ideas in the intact rat using a threefold approach. First, we asked whether sniff strength shapes odor representations in vivo by imaging from olfactory receptor neuron (ORN) terminals during controlled changes in inhalation flow in the anesthetized rat. Second, we asked whether sniff strength shapes odor representations by imaging from ORNs during natural sniffing in the awake rat. Third, we asked whether rats actively modulate sniff strength during an odor discrimination task. We found that, while artificial changes in flow rate can alter ORN responses, sniff strength has negligible effect on odor representations during natural sniffing, and behaving rats do not modulate flow rate to improve odor discrimination. These data suggest that modulating sniff strength does not shape odor representations sufficiently to be part of a strategy for active odor sensing in the behaving animal.

\section{Introduction}

Sensation depends on the acquisition of sensory information and active sensory sampling during behavior shapes the neural representation, central processing and, ultimately, perception of an animal's environment. Examples of active sampling include tactile finger movements, whisking in rodents, and saccades in vision. Active sampling is particularly important in olfaction, where phylogenetically diverse animals intermittently sample their olfactory environment with behaviors that are qualitatively similar (Dethier, 1987; Wachowiak, 2011). In terrestrial vertebrates, including mammals, olfactory sensation depends on inhalation in the course of respiration or active sniffing. A "sniff" constitutes the basic unit of odor sensing and is fundamental to how olfactory information is represented and processed (Mainland and Sobel, 2006; Uchida et al., 2006). Because animals precisely control multiple parameters of sniffing during behavior

\footnotetext{
Received Aug. 26, 2012; revised 0ct. 3, 2012; accepted 0ct. 24, 2012

Author contributions: T.C. and M.W. designed research; T.C., J.P.M., Y.T., and J.V.V. performed research; J.V.V. contributed unpublished reagents/analytic tools; T.C., J.P.M., and M.W. analyzed data; T.C. and M.W. wrote the paper.

This work was supported by funding from the National Institutes of Health (DC06441 and DC011423). We thank K. Zhao and J. Scott for useful discussions on odorant sorption dynamics, D. Katz for advice on behavioral paradigms, and M. Hasselmo for additional support.

The authors declare no competing financial interests.

Correspondence should be addressed to Matt Wachowiak, Department of Neurobiology and Anatomy, University of Utah, Salt Lake City, UT 84103. E-mail: matt.wachowiak@utah.edu

DOI:10.1523/JNEUROSCI.4101-12.2013

Copyright $\odot 2013$ the authors $\quad 0270-6474 / 13 / 330079-14 \$ 15.00 / 0$
}

(Youngentob et al., 1987; Kepecs et al., 2007; Wesson et al., 2008b), understanding the relationship between sniffing and olfactory information processing is critical for understanding olfactory function.

Perhaps the earliest hypothesis of the role of sniffing in olfaction relies on the observation that odorants with different physicochemical properties exhibit different patterns of deposition across the olfactory epithelium, and that these patterns are differentially affected by flow rate (Adrian, 1950; Mozell, 1964, 1970; Mozell and Jagodowicz, 1973; Scott et al., 2006; Yang et al., 2007b). This hypothesis, which we term the sorption hypothesis, provides a potential strategy for actively shaping odor representations by modulating inhalation flow rate, or sniff strength, during behavior. The sorption hypothesis predicts that odorant sampling may be actively controlled to optimize the detection or discrimination of odorants with different sorption properties in olfactory mucosa (Youngentob et al., 1987). In particular, fluid dynamic modeling studies of the rat and human nasal cavity predict that increasing inhaled flow rate leads to increased deposition of odorants with high solubility in mucosa (strongly sorbed odorants) and little change or a decrease for weakly sorbed odorants (Mozell et al., 1987; Hahn et al., 1994).

Flow-rate effects consistent with the sorption hypothesis have been measured from the olfactory epithelium in rodents (Kent et al., 1996; Scott-Johnson et al., 2000) and from the olfactory bulb using artificial inhalation (Oka et al., 2009). Such effects remain untested during natural odor sampling, however. In addition, behavioral evidence that animals actively modulate sniff strength 
depending on odorant sorption properties remains lacking (but see Rojas-Libano and Kay, 2012). Here, we tested the sorption hypothesis in the context of natural odor sampling and in the awake animal. We used a threefold approach. First, using controlled changes in inhalation flow over a behaviorally relevant range in the anesthetized rat, we asked whether flow rate shapes olfactory receptor neuron (ORN) response patterns in vivo. Second, we asked whether sniff strength shapes ORN responses during natural sniffing in the awake rat. Finally, we asked whether rats actively modulate sniff strength for odorants with different sorption properties during an odor discrimination task. Taken together, these experiments confirm that odorant sorption properties have the potential to shape ORN responses but suggest that during natural sniffing in the awake animal the importance of odorant sorption, and of sniff strength in general, is surprisingly limited.

\section{Materials and Methods}

Adult female Long-Evans rats (body weight, 220-280 g) were used in all experiments. All procedures were approved by the Institutional Animal Care and Use Committees of Boston University and the University of Utah and were in accordance with guidelines established by the United States National Institutes of Health. All statistics report mean \pm SD unless otherwise noted. Tests for normality were performed on all data before statistical analysis and nonparametric tests were used for data not fitting a normal distribution. Parametric tests were unpaired, twosample $t$ tests unless stated otherwise. All statistical tests were performed using functions in Matlab or Origin.

Surgical procedures. All surgical procedures have been previously described in detail (Verhagen et al., 2007; Carey and Wachowiak, 2011). Briefly, for imaging in anesthetized rats, animals were anesthetized with a mixture of urethane and chlorprothixene, a double tracheotomy was performed to allow for artificial control of inhalation independent of free respiration, and the bone overlying both olfactory bulbs was exposed and thinned for imaging. For head-fixed imaging, in an initial procedure using isoflurane anesthesia, a guide cannula (PlasticsOne) was implanted in the dorsal recess of one naris for later intranasal thermocouple measurements and a head bolt was implanted on the skull for restraint. A dummy cannula prevented occlusion of the guide cannula between subsequent behavioral sessions. After behavioral training (see below), the bone overlying each olfactory bulb was thinned and a chronic optical window installed under isoflurane anesthesia. For both sets of imaging experiments, ORNs in both nares were loaded with the dextranconjugated calcium-sensitive dye Oregon Green 488 BAPTA-1 dextran (molecular weight, $10 \mathrm{kDa}$; Invitrogen) 3-5 d before the first imaging session (Verhagen et al., 2007).

Behavioral training. Animals were trained to accept head fixation and to perform operant tasks using water restriction for motivation and followed the training sequence described previously (Verhagen et al., 2007; Wesson et al., 2009). In the awake imaging experiments, rats were trained either to perform a lick/no-lick two-odor discrimination task for water reward with $2 \%$ sucrose, or simply licked for intermittent water reward and were passively exposed to odor (see Fig. $3 A$ ). For the experiments testing behavioral modulation of inhalation flow rate (see Fig. 7), rats were trained to perform a two-odor discrimination using a pair of structurally similar odorants and, after successful discrimination performance in two consecutive sessions, odorant concentration was incrementally reduced until performance fell below $80 \%$ correct over two consecutive blocks of trials. Rewarded ( $\mathrm{S}+$ ) versus unrewarded $(\mathrm{S}-$ ) odorant trials were counterbalanced within each trial block ( 25 or 50 trials/block).

Optical recordings. Calcium signals were imaged in head-fixed rats using a custom-built imaging apparatus with Olympus epifluorescence optics (BX51). The olfactory bulb (OB) was illuminated with full light from a $150 \mathrm{~W}$ xenon arc lamp (Opti-Quip) and visualized with the following filter set: HQ480/40 (excitation), Q505LP (dichroic), and HQ535/50 (emission). A region of the dorsal OB spanning $2.8 \pm 0.2 \mathrm{~mm}$ in the rostrocaudal dimension and $1.5 \pm 0.1 \mathrm{~mm}$ in the mediolateral
Table 1. Sorption properties of odorants used

\begin{tabular}{|c|c|c|c|c|}
\hline Odorant & Uptake $^{a}$ & $\begin{array}{l}\text { Retention } \\
\text { time }^{b}\end{array}$ & $\begin{array}{l}\text { Partition } \\
\text { coefficient }^{c, d}\end{array}$ & $\begin{array}{l}\text { Henry's } \\
\text { law constant }\end{array}$ \\
\hline \multicolumn{5}{|c|}{ Strongly sorbed odorants } \\
\hline Methyl benzoate & 0.9 & 1 & * & $1.3 \mathrm{e} 3$ \\
\hline Benzaldehyde & 0.84 & 0.8 & $*$ & 870 \\
\hline Isovaleric acid & * & 10 & $2.9 \mathrm{e} 4$ & $2.5 \mathrm{e} 5$ \\
\hline Menthone ${ }^{e}$ & * & $*$ & * & 210 \\
\hline \multicolumn{5}{|c|}{ Weakly sorbed odorants } \\
\hline Heptanal & 0.32 & 0.1 & $9.1 \mathrm{e} 1$ & 72 \\
\hline Propyl acetate ${ }^{f}$ & * & * & * & 110 \\
\hline Amyl acetate & 0.2 & 0.1 & $4 \mathrm{e} 2$ & 56 \\
\hline Butyl acetate & $*$ & 0.1 & $6.2 \mathrm{e} 2$ & 77 \\
\hline Methyl valerate & * & * & * & 59 \\
\hline
\end{tabular}

Values show different measures of sorption properties for odorants used. The first three columns show different experimental measurements of odorant sorption, measured as uptake (fraction removed from airstream after passage through human nasal cavity), retention time (relative retention time of odorant by frog olfactory epithelium) or partition coefficient (relative partitioning of odorant into water vs air ${ }^{c}$ or mucus vs air ${ }^{d}$ ). The fourth column shows water-air partition coefficients calculated as Henry's law constants using the group method in HENRYWIN 3.2 (Environmental Protection Agency) at $25^{\circ} \mathrm{C}$. Higher numbers reflect stronger odorant sorption.

${ }^{a}$ Kurtz et al., 2004.

${ }^{b}$ Mozell and Jagodowicz, 1973.

'Amoore and Buttery, 1978.

${ }^{d}$ Hornung and Mozell, 1981; Hornung et al., 1987b.

${ }^{e}$ Yang et al., 2007b.

Kent et al., 1996

*Values not available from experimental literature.

dimension was imaged using a $4 \times, 0.28$ numerical aperture objective (Olympus). Images were acquired with a $256 \times 256$ pixel CCD camera (NeuroCCD; SM-256, RedShirtImaging) at 25 or $100 \mathrm{~Hz}$ frame rates. The camera resolution was $13.1 \mu \mathrm{m}$ per pixel. The anterior and posterior edges of the imaged region were at $\sim 9.9$ and $7.1 \mathrm{~mm}$ anterior to bregma, respectively, and the medial and lateral edges at 0.3 and $1.8 \mathrm{~mm}$ lateral to midline, respectively. Records of respiration, licking, and odorant delivery were synchronized with the optical signals and saved at $100 \mathrm{~Hz}$ in Neuroplex.

Olfactometry. Odorants were simple monomolecular compounds and were nominally classified as either strongly or weakly sorbed based on prior experimental measurements of odorant sorption by olfactory tissue (Table 1). All odorants were purchased from Sigma-Aldrich or Fisher Scientific. For presentation, odorants were diluted to $1 \%$ with clean air from the saturated vapor of liquid odorant. Liquid odorant was pure for the imaging experiments and was diluted by 1:10 to 1:10,000 in dipropylene glycol for the inhalation behavior experiments. Liquid dilutions for the odorant pairs were balanced so that the saturated vapor concentration was near equivalent for each odorant in the pair $(\sim 4 \mathrm{ppm}$ at the initial training concentration).

Measurement and control of odor sampling. Inhalation in awake rats was measured via the intranasal cannula using either a pressure sensor (model 24PCAFA6G, Honeywell) or by inserting a thermocouple (model emtss-010 g-12, Omega Engineering) into the dorsal recess, as described previously (Verhagen et al., 2007). Thermocouple and pressure signals were amplified $(100-1000 \times)$, low-pass filtered at 50 or $100 \mathrm{~Hz}$, digitized at $500 \mathrm{~Hz}$, and saved to disk. Artificial inhalation and "sniff playback" in anesthetized rats were performed after blocking one naris to ensure controlled airflow through the side ipsilateral to the imaged OB. Sniff playback was performed as described previously (Cheung et al., 2009; Carey and Wachowiak, 2011) using command waveforms derived from intranasal pressure measurements in awake, head-fixed rats. The "low-flow" and "high-flow" sniff waveforms were generated by averaging 20 pressure transients (i.e., "sniffs") that resulted in small and large intranasal flow rates, as measured simultaneously using a thermocouple (see Fig. $2 A$ ). Exhalation components preceding inhalation were omitted from the command waveform. During the experiment, the gain of the sniff playback device was adjusted so that the low-flow sniff evoked ORN responses similar in amplitude to those evoked by short-duration (250 $\mathrm{ms})$ and moderate flow-rate $(250 \mathrm{ml} / \mathrm{min})$ artificial inhalation. Peak deflection of the sniff playback syringe approximated rat tidal volume (1.4 
A
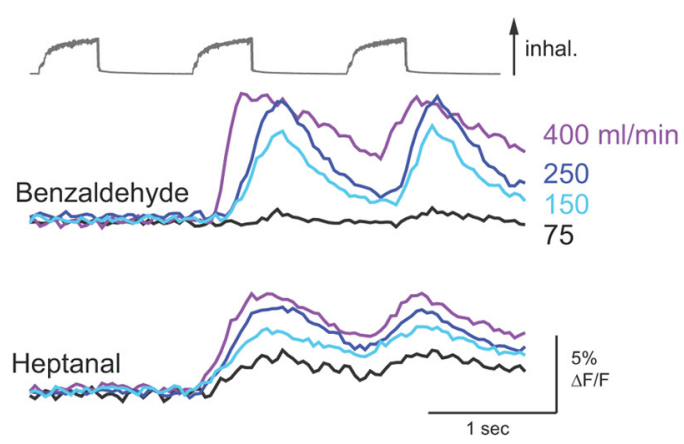

C

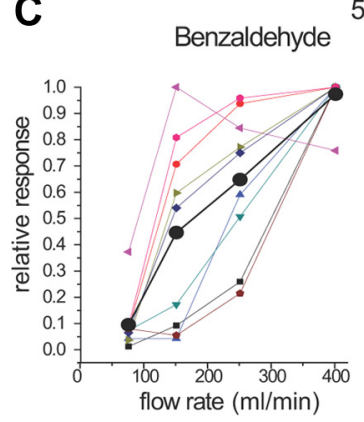

\section{$\mathbf{E}$}

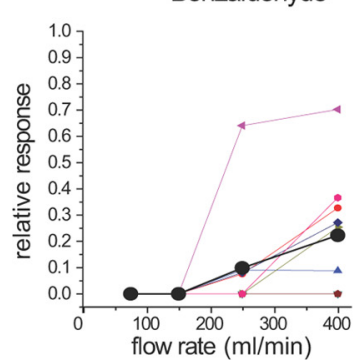

$500 \mathrm{~ms}$ inhalation

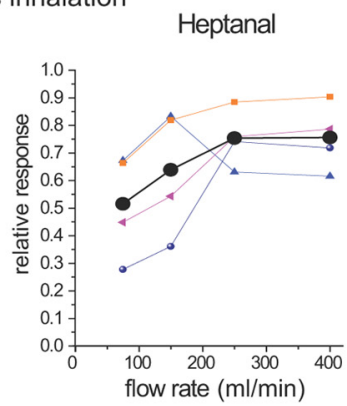

100 ms inhalation

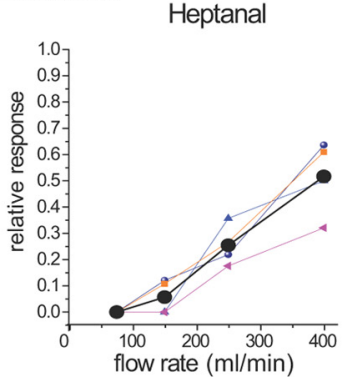

B

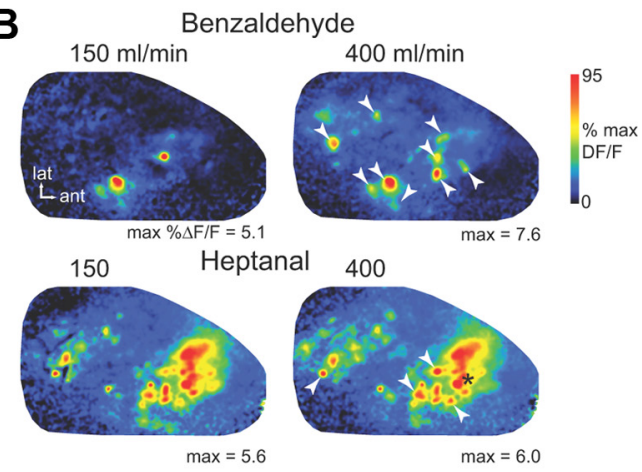

D

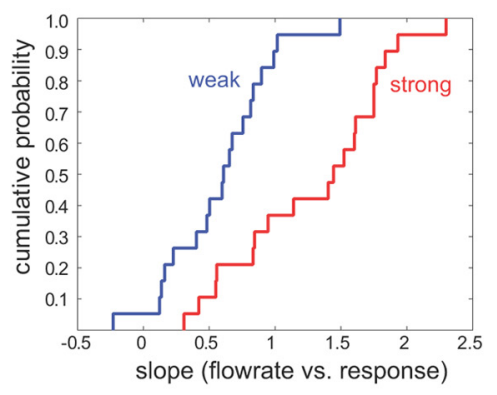

$\mathbf{F}$

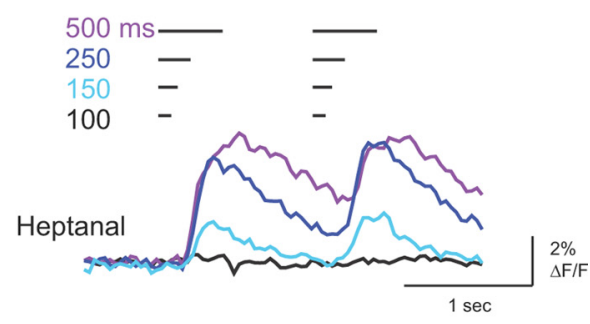

Figure 1. Odorant sorption affects flow-rate dependence of ORN responses during artificial inhalation. $A$, Presynaptic calcium signals imaged from ORNs in vivo during artificial inhalation of a strongly sorbed (benzaldehyde) and weakly sorbed (heptanal) odorant. Traces show calcium signal taken from one glomerulus during inhalation at increasing flow rates (indicated by numbers at right). Glomeruli are different for each odorant. Increasing flow rate leads to increased peak amplitude and decreased latency of responses. $\boldsymbol{B}$, Peak response maps from the same preparation evoked by inhalation of each odorant at 150 and $400 \mathrm{ml} / \mathrm{min}$. Each map is normalized to its own maximum. For each odorant, increasing flow rate activates additional glomeruli in the map. Arrowheads indicate glomeruli plotted in $\boldsymbol{C}$; asterisk indicates glomerulus shown in $\boldsymbol{F}$. C, Flow rate-response relationships plotted for multiple glomeruli activated by each odorant (same preparation as in $\boldsymbol{A}$ and B). Thick black plot shows mean response across activated glomeruli. Note that slope appears shallower for heptanal than for benzaldehyde. Response axis normalized to maximal response for each glomerulus. D, Cumulative probability distribution for normalized flow rate-response slopes for all glomeruli, plotted for weakly and strongly sorbed odorants. $\boldsymbol{E}$, Flow rate-response plots for 0 RN inputs to the same glomeruli in the same preparation, but using shorter-duration $(100 \mathrm{~ms})$ inhalations. At this duration, the lowest flow rate $(75 \mathrm{ml} / \mathrm{min})$ fails to evoke inputs to any glomeruli. Also heptanal flow rate-response slopes are steeper and appear more linear than for longer-duration ( $500 \mathrm{~ms}$ ) inhalations, indicating less saturation of 0 RN responses (see $\boldsymbol{C}$ ). $\boldsymbol{F}$, (alcium signals from one glomerulus ( $\boldsymbol{B}$, asterisk) showing responses to inhalations ranging in duration from 100 to $500 \mathrm{~ms}$, using the lowest flow rate. Responses are nearly undetectable for $100 \mathrm{~ms}$ duration and begin to show saturation between 250 and 500 ms duration.

$\mathrm{ml})$. Actual pressure transients generated during sniff playback were recorded in parallel with each trial for later normalization of ORN responses with respect to sniff strength (see below). Simple artificial inhalation pulses (duration, 100-500 ms; Fig. 1) were generated using a three-way solenoid valve that gated a vacuum line set at different flow rates $(75-600 \mathrm{ml} / \mathrm{min})$. Fidelity of the artificial inhalation pulses was also monitored online using a pressure sensor.

Inhalation flow-rate measurements. Relative inhalation flow rates in awake rats were estimated using two measures, one directly from the thermocouple signal and the other derived from the intranasal pressure signal. Thermocouple signals were digitally filtered from 0.01 to $20 \mathrm{~Hz}$ (second-order Butterworth) to reduce measurement noise. Inhalations were identified using an automated peak detection algorithm in Matlab or LabView and peak flow defined as the amplitude of this peak relative to its preceding trough. Previous measurements of thermocouple signals obtained with an identical setup under conditions of controlled intrana- sal flow confirmed that the peak thermocouple signal amplitude scaled linearly with peak flow rate (Cheung et al., 2009). Pressure signals were low-pass filtered at $50 \mathrm{~Hz}$ (with no high-pass filtering) and mean inhalation flow rate estimated as $V_{\mathrm{i}} / t_{\mathrm{i}}$ where $V_{\mathrm{i}}$ is the volume of air inhaled, estimated as the area under the negative pressure component of the pressure transient and $t_{\mathrm{i}}$ the duration of inhalation (see Fig. 6A). $V_{\mathrm{i}}$ and $t_{\mathrm{i}}$ were measured from the beginning of inhalation ( 0 -crossing) to the time of decay back to $25 \%$ of peak inhalation pressure. For both measures, an operational range of inhalation flow rates was defined as from the $3 \mathrm{rd}$ percentile to the 97th percentile of measurements for all inhalations (out of 3000-6000 per session) occurring with an intersniff interval (ISI) of $>400 \mathrm{~ms}$. Inhalation amplitudes were normalized to this operational range for correlation with ORN-response amplitudes.

Optical signal analysis. ORN-response magnitudes were measured from optical signals extracted from regions of interest (ROIs) representing responsive glomeruli. ROIs were chosen manually using criteria 
based on signal-to-noise ratio and size (Verhagen et al., 2007). For the artificial inhalation and sniff playback experiments, responses were measured from the peak of the first inhalation after odorant presentation, relative to the signal just before inhalation $(\Delta F)$. Responses were measured from four-trial averages using the temporal average of four frames (160 ms) at both peak and baseline and normalized to the resting fluorescence (percentage $\Delta F / F$ ).

For the awake imaging experiments, optical signals were first corrected for small movement-related artifacts and high-pass spatial filtered to reduce global hemodynamic signals and then ROIs identified and extracted for further analysis using custom software written in Matlab (Verhagen et al., 2007). Signals were upsampled to $100 \mathrm{~Hz}$ to match the respiration signals and inhalation-evoked ORN-response amplitudes measured by fitting the signal from each ROI to a double-sigmoid function (Wesson et al., 2008a). To avoid confounds of receptor adaptation, ORN responses from awake rats were analyzed only for the first inhalation occurring at least $50 \mathrm{~ms}$ after odorant onset (to ensure that odorant concentrations at the naris reached a steady-state level) and for later inhalations occurring with an ISI of at least $2 \mathrm{~s}$ (this was rare). Trials in which inhalations occurred before the 50 ms cutoff, or in which a second inhalation occurred within $200 \mathrm{~ms}$, were excluded from analysis.

Flow-response slopes were measured from a linear fit to each ROI/ odorant pair. To avoid pseudoreplication in cases where the same ROI was imaged for the same odorant in multiple sessions, normalized response amplitudes and peak flow measurements were combined across sessions (maximum 2-3 sessions) before fitting. To compare low-flow and high-flow inhalations in awake animals, responses corresponding to inhalations generating flows within the lower or upper $25 \%$ of values were averaged. Response maps for low-flow and high-flow inhalations were compared quantitatively using the mean difference (MD) across all ROIs in a session, calculated as $\left\langle\left|\Delta F_{x} / F_{x}-\Delta F_{y} / F_{y}\right|\right\rangle$, where $x$ and $y$ indicate the response in the same ROI to low-flow and high-flow inhalations (high vs low MD) or to different inhalations of the similar strength (self MD) (Verhagen et al., 2007). To enable comparison of MD values from sessions and animals with variable levels of loading, MD values were normalized to the peak evoked response amplitude in each session; thus $\mathrm{MD}$ values represent a fraction of the odorant-evoked dynamic range of responses.

Behavioral signal analysis. In the sorption/odorant discrimination experiments, peak inhalation flow rates were measured from thermocouple signals and normalized ( $z$-scaled) to the mean and SD of all inhalations in the $5 \mathrm{~s}$ preodorant interval for all trials in a session. To test for changes in inhalation flow rate across trials, only inhalations occurring after odorant onset and before the median odorant response time were used. Odorant response time was calculated separately for each block of 25 or 50 trials and defined as the median of the time from odorant onset to first lick for all S+ trials in that block. Typically there were 1-3 inhalations in this time window. When several inhalations occurred in this window, flow rate was measured as the average value of each inhalation; in case none occurred, flow rate was measured from the first inhalation after odorant onset. Potential effects of dilution and sorption on flow rate were analyzed with ANOVA.

\section{Results}

We sought to test longstanding predictions on the relationship between inhalation flow rate, odorant sorption properties, and ORN responses in the awake animal. Specifically, the two key predictions relevant to active control of peripheral odor representations are (1) that increasing inhalation flow rate will lead to increased activation of ORNs for strongly sorbed odorants and (2) that increasing inhalation flow rate will lead to little or no change in ORN activation for weakly sorbed odorants. Because these predictions have been developed in the greatest detail in the rat (Hahn et al., 1994; Kent et al., 1996; Yang et al., 2007b), we used rats in all experiments.

We tested these predictions by imaging action potentialevoked calcium influx into ORN axon terminals at their convergence onto OB glomeruli (Wachowiak and Cohen, 2001;
Verhagen et al., 2007; Oka et al., 2009). This approach reflects ORN spiking activity, allows for monitoring multiple distinct ORN populations defined by odorant sensitivity, reports activity on a temporal scale matching that of inhalation, and can report activity in the intact and behaving animal. Presynaptic calcium signals are also graded in amplitude, with increases in peak amplitude reflecting increases in evoked ORN spiking activity across the population of neurons converging onto one glomerulus. The fidelity of these signals is sufficient to report modulation of ORN responsiveness by modest increases in odorant concentration as well as by other changes in sampling behavior, such as sniff frequency (Wachowiak and Cohen, 2001; Verhagen et al., 2007; Carey et al., 2009).

\section{Flow rate-sorption relationships measured using artificial inhalation}

Previous studies from rats have reported significant effects of flow rate on the magnitude and patterns of ORN activation in excised epithelium preparations (Kent et al., 1996), consistent with the sorption hypothesis. To test this in vivo, we imaged responses from ORN terminals in anesthetized rats using artificial inhalation pulses of different flow rates and duration (see Materials and Methods). Flow rates varied from 75 to $600 \mathrm{ml} / \mathrm{min}$ (through one nostril), spanning the range of peak inhalation rates reported for awake rats (Youngentob et al., 1987). Odorants were chosen based on their ability to evoke robust input to glomeruli in the dorsal $\mathrm{OB}$ and designated as either strongly or weakly sorbed based on previous empirical studies measuring odorant uptake in the nasal cavity or on calculated water-air partition coefficients (see Materials and Methods; Table 1).

Because existing predictions of sorption-dependent flow-rate effects arise largely from modeling steady-state airflow patterns (Hahn et al., 1994; Yang et al., 2007b), we first used long (500 ms) inhalation pulses in an attempt to approximate such conditions experimentally, despite the fact rats never express such long inhalations (Hegoburu et al., 2011). An example of ORN responses evoked by artificial inhalation of a strongly and a weakly sorbed odorant, imaged from the same preparation, is shown in Figure $1 A$. Responses rise rapidly and peak within several hundred milliseconds after inhalation onset; for at least some glomeruli, ORN responses begin to decay before the end of the inhalation pulse, indicating rapid adaptation of the ORN response. The temporal dynamics of the inhalation-evoked response differed for different odorants and glomeruli as described previously (Spors et al., 2006; Carey et al., 2009) (Fig. 1A), but dynamics did not differ systematically between strongly and weakly sorbed odorants. Increasing inhalation flow rate most often led to increases in ORNresponse amplitude as well as a decrease in response latency (Fig. $1 A$ ), although this relationship was not uniform across different ORN populations in the same preparation. As a result, patterns of ORN input to different glomeruli (i.e., odorant response "maps") could change as flow rate increased (Fig. $1 B$ ), consistent with earlier observations in the mouse olfactory bulb (Oka et al., 2009).

Increases in ORN-response amplitude and changes in relative response patterns do not directly address predictions from the sorption hypothesis, as the molar increase in odorant deposition at higher flow rates would be predicted to increase ORN responses and to recruit ORN inputs to additional glomeruli (Duchamp-Viret et al., 1999; Malnic et al., 1999; Wachowiak and Cohen, 2001; Oka et al., 2009). To address the sorption hypothesis more directly, we tested for systematic differences in the relationship between flow rate and ORN responses for weakly 
versus strongly sorbed odorants ( $n=5$ animals). Specifically, we tested whether this relationship was stronger for strongly sorbed odorants than for weakly sorbed odorants. Strongly sorbed odorants were benzaldehyde ( $n=17$ glomeruli, 3 animals) and methyl benzoate ( $n=2$ glomeruli, 1 animal); weakly sorbed odorants were heptanal ( $n=17$ glomeruli, 3 animals) and butyl acetate ( $n=2$ glomeruli, 1 animal).

Flow rate-response relationships were quantified for responsive glomeruli (total, 38 glomeruli) using linear regression. Linear regression, as opposed to fitting to a sigmoid function, was used because of the relatively small number of flow rates (typically 4) tested per experiment. To enable comparison of flow rate-response slopes across glomeruli and to avoid confounds due to response saturation of the most sensitive ORNs, each odorant was also tested at twice the working concentration ( $0.5-1 \%$ saturated vapor) to define a "maximal" response, and response amplitudes for the different flow rates normalized to this response (Fig. 1C). Glomeruli showing responses $>70 \%$ of the maximal response at the lowest flow rate tested were excluded from analysis because of potential saturation even at the lowest concentrations. Slopes of the flow rate-response relationship were significantly higher for strongly sorbed than for weakly sorbed odorants (Fig. 1D) (strong: mean, $1.29 \pm 0.6, n=19$; weak: mean, $0.59 \pm 0.4, n=19 ; p<0.001)$. Because ORNresponse magnitudes are more strongly dependent on inhalation flow rate for strongly sorbed odorants than for weakly sorbed odorants, these results are consistent with predictions from the sorption hypothesis.

Because odorant sorption into and clearance of odorant from the epithelium are time-dependent processes, we decreased the duration of artificial inhalation in order to test whether the flow rate-dependent effects observed reflected steady-state levels of odorant sorption. Figure $1 E$ shows flow rate effects for the same odorants and glomeruli as in Figure $1 C$ using inhalation durations of $100 \mathrm{~ms}$, which are in the range of inhalation durations in awake, freely breathing rats (Roux et al., 2006; Hegoburu et al., 2011). Figure $1 F$ shows responses from one glomerulus evoked by different duration inhalations at a minimal flow rate $(75 \mathrm{ml} / \mathrm{min})$. Shortening inhalation duration typically led to smaller ORN responses and could significantly alter the relationship between flow rate and response magnitudes from that seen with longer durations. At lower flow rates, inhalations of 100-150 ms often failed to elicit any responses, suggesting that such inhalations were insufficient to draw odorant into the olfactory region of the nasal cavity (Fig. $1 F$ ). In contrast, increasing duration from 250 to $500 \mathrm{~ms}$ typically led to little increase in response amplitude (Fig. $1 F$ ), consistent with responses reaching steady state by $250-500 \mathrm{~ms}$ but not by $100-150 \mathrm{~ms}$. Slopes of flow rate-response relationships measured for 250-ms-duration inhalations were not significantly different from those measured for $500 \mathrm{~ms}$ inhalations [mean slope for weakly sorbed odorants: $0.68 \pm 0.29, n=14, p=0.4$ vs $500 \mathrm{~ms}$ inhalations; median slope for strongly sorbed odorants: 1.44 for $250 \mathrm{~ms}$ $(n=3)$ and $500 \mathrm{~ms}(n=19)$ inhalations]. It was not possible to obtain slopes for 100-ms-duration inhalations because of the failure of many glomeruli to respond at the lower flow rates. These results suggest that the dynamics of odorant sorption and its relationship to flow rate during natural odor sampling may differ from those observed under steady-state conditions.
Flow rate-sorption relationships measured using naturalistic sniff waveforms

Awake behaving animals dynamically modulate intranasal airflow by changing the magnitude, duration, and frequency of respiratory pressure transients (Fig. 2A). To explore the effect of flow rate on ORN responses evoked by naturalistic samples of odorant, we used a "sniff playback" device capable of accurately reproducing naturalistic odor sampling behavior (Carey et al., 2009; Cheung et al., 2009). We used sniff playback to sample odorants using intranasal pressure transients ("sniffs") that generated either low or high peak intranasal flow rates $(n=5$ animals). The two (low-flow and high-flow) command waveforms were generated from pressure measurements recorded from an awake, head-fixed rat in which intranasal airflow signals were simultaneously measured via a thermocouple (Fig. $2 A, B$ ). We confirmed that subsequent playback of these command waveforms in anesthetized rats also generated high and low peak intranasal flow rates as measured with a thermocouple (Fig. 2B). The low-flow and high-flow "sniffs" had durations of 140 and 100 ms respectively, at half-maximal amplitude, and 250 and $180 \mathrm{~ms}$ at their base.

Figure $2 B$ shows responses evoked by low-flow and high-flow rate "sniffs" of the same odorant. As with artificial inhalation, higher flow-rate sniffs typically led to larger ORN responses. In contrast to artificial inhalation, higher flow-rate sniffs led to little change in ORN response dynamics (Fig. 2B). Consistent with their shorter duration, sniff playback evoked input to fewer glomeruli than $500 \mathrm{~ms}$ inhalation pulses for the same odorants (Fig. 2C). Nonetheless high-flow sniffs recruited inputs to more glomeruli than for low-flow sniffs, leading to a change in response maps (Fig. 2C). Using the change in peak inhalation pressures measured during playback of low-flow and high-flow sniffs, we compared the strength of the relationship between sniff flow rate and ORN-response magnitude for weakly and strongly sorbed odorants. Strongly sorbed odorants were benzaldehyde ( $n=5$ glomeruli, 2 animals), methyl benzoate ( $n=9$ glomeruli, 2 animals), and menthone ( $n=1$ glomerulus, 1 animal); weakly sorbed odorants were heptanal ( $n=13$ glomeruli, 2 animals), butyl acetate ( $n=3$ glomeruli, 1 animal), and propyl acetate $(n=$ 5 glomeruli, 1 animal). This relationship was weaker than in the artificial inhalation experiments (strongly sorbed slopes: mean, $0.80 \pm 0.4 ; n=15$ glomeruli; 5 animals; weak: mean, $0.43 \pm 0.3$; $n=21$ glomeruli; 4 animals). Nonetheless changing from "lowflow" to "high-flow" sniffs led to a stronger increase in ORN responses for strongly sorbed than for weakly sorbed odorants (Fig. $2 D)(p<0.01)$. These results are also consistent with predictions of flow rate-ORN response relationships arising from the sorption hypothesis.

\section{Limited correlation between sniff strength and ORN responses in the awake rat}

The sniff playback experiments tested inhalation waveforms that represented the low and high end of the range of flow rates generated during natural odorant sampling, but required an estimate of absolute flow rates and could not capture the diversity of sampling parameters exhibited in the awake, behaving rat (Youngentob et al., 1987). Having established the ability to detect relationships between flow rate and ORN responses in vivo, we next designed experiments to test whether such relationships persist in awake rats as they naturally sample odorants. In these experiments, we imaged ORN responses from the dorsal OB of awake, head-fixed rats performing a simple operant task (Verhagen et al., 2007). In one variant of the task (3 animals; 7 total 
A

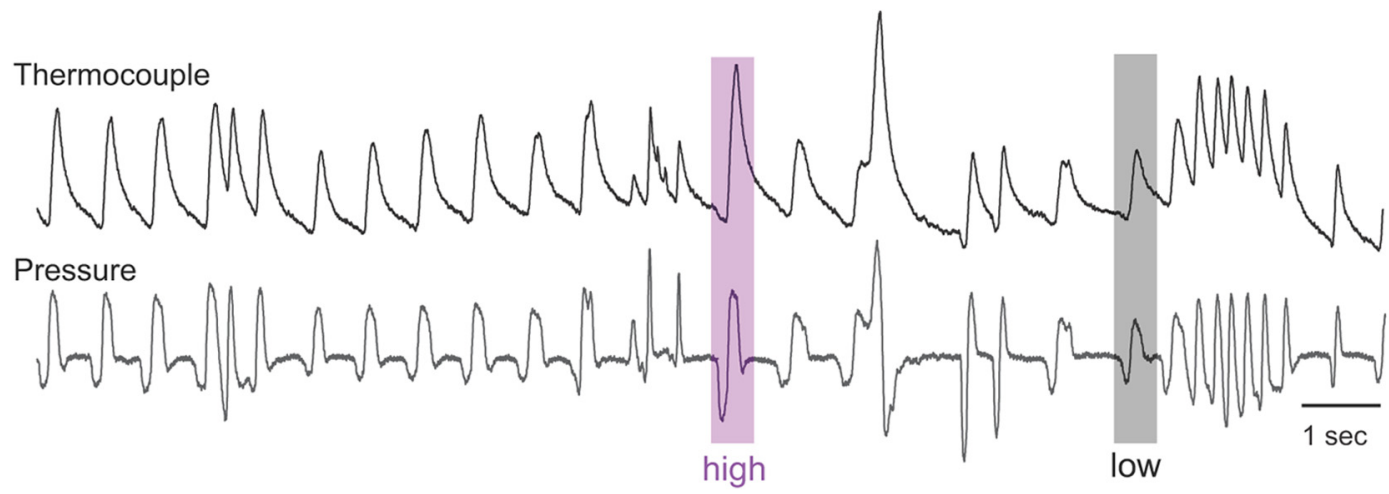

B
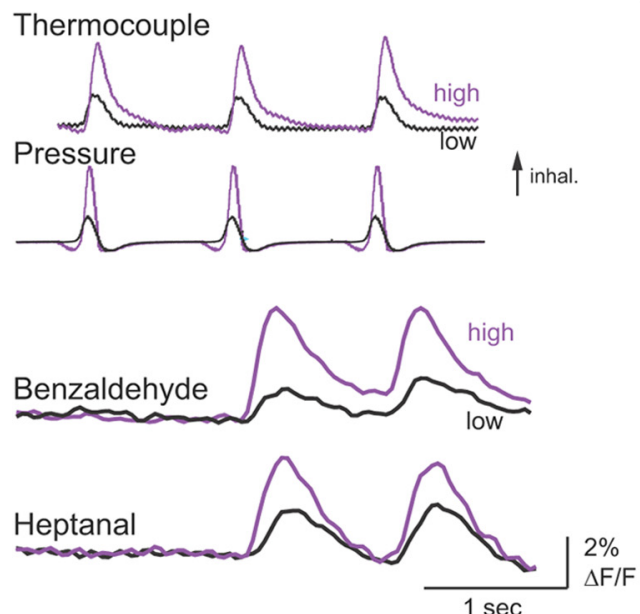

C
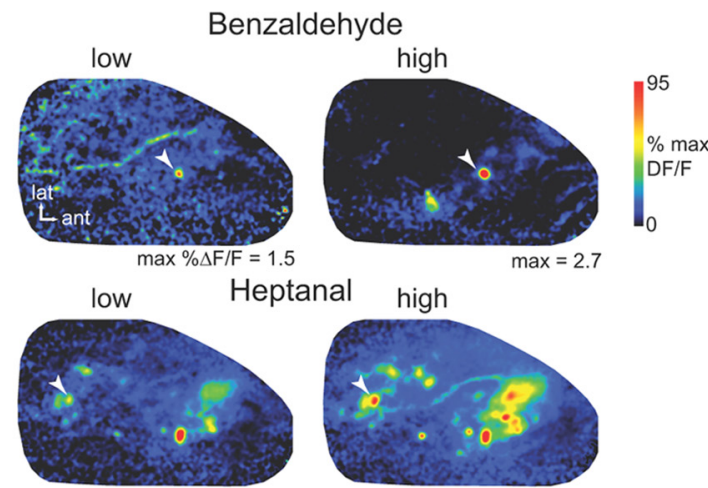

$\max =4.1$

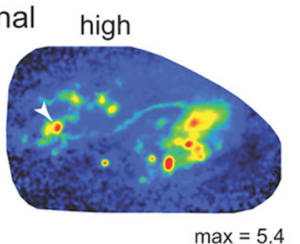

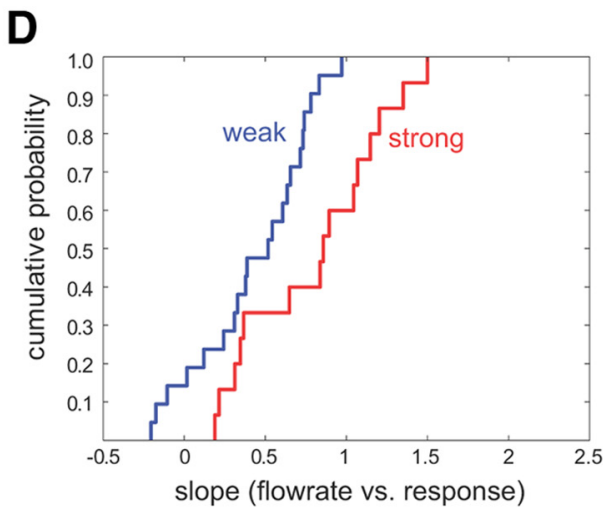

Figure 2. Sorption effects are weaker during playback of naturalistic sniffs in the anesthetized rat. $A$, Derivation of low and high flow rate "sniffs" from the awake rat. Traces show simultaneous measurements of intranasal airflow measured via a thermocouple (top trace) and intranasal pressure (lower trace) measured through the same cannula. Inward airflow and negative pressure (inhalation) are up. Purple and gray shaded boxes indicate inhalations generating low and high peak flow rates as measured via thermocouple. The corresponding pressure traces were used as command waveforms for sniff playback. See Materials and Methods for details. These boxes illustrate examples only; actual waveforms are shown in $\boldsymbol{B}$. $\boldsymbol{B}$, Presynaptic calcium signals from ORN input to a glomerulus evoked by playback of the low and high flow rate "sniffs" for benzaldehyde and heptanal. Top traces show intranasal airflow (thermocouple) and pressure measured during playback. Same animal as for artificial inhalation in Figure $1 A-C$.C, Peak response maps (same preparation) for both odorants, evoked by playback of low and high flow rate sniffs. Each map is normalized to its own maximum. For each odorant, the stronger sniff evokes input to more glomeruli and with increasing amplitude. Arrowheads indicate glomeruli plotted in $\boldsymbol{B}$. Note that sniff playback evokes input to many fewer glomeruli than does $500 \mathrm{~ms}$ artificial inhalation (compare with Fig. 1B, maps). D, Cumulative probability distribution for flow rate-response slopes derived from the two sniff strengths (see Results, Materials and Methods for details) for all glomeruli, plotted for weakly and strongly sorbed odorants.

sessions), rats were trained to perform a go/no-go two-odor discrimination task in exchange for water reward. In the other variant (2 animals; 5 sessions), rats were trained to simply lick for water reward presented at random intervals and odor (not associated with reward) was presented during the interval between rewards (Fig. $3 A$ ). In each task, at least one strongly sorbed and one weakly sorbed odorant were presented while imaging optical signals from the dorsal OB. To correlate ORN-response magni- tudes with intranasal flow rate on a sniff-by-sniff basis, we simultaneously measured changes in flow rate in the dorsal nasal cavity using a thermocouple. Inhaled air led to cooling of the thermocouple, and the amplitude of the deflection was proportional to the peak inhalation flow rate (Cheung et al., 2009).

Head-fixed rats showed respiratory patterns that were highly variable over time. Peak inhalation flow rate, or sniff amplitude, as reflected in the thermocouple signal, varied substantially 


\section{A i. Two-odor discrimination} Session and choice structure $\mathrm{S}+\ldots \mathrm{S}-\mathrm{a} \ldots \mathrm{S}+\ldots \mathrm{S}-{ }_{b} \ldots \mathrm{S}+\ldots$ etc.

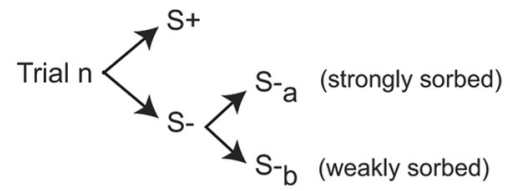

\section{ii. Passive odorant presentation}

\section{Trial structure}

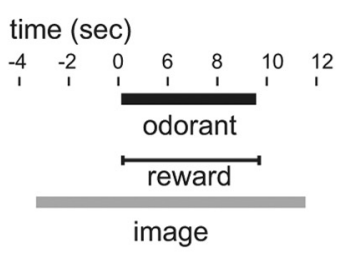

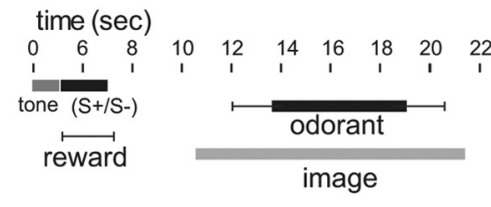

B

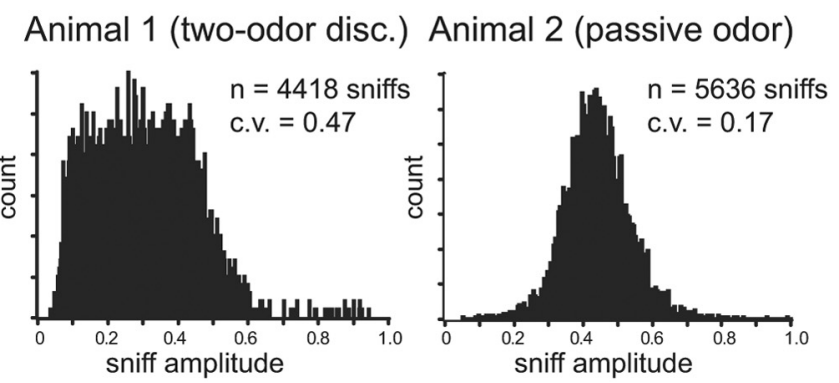

C

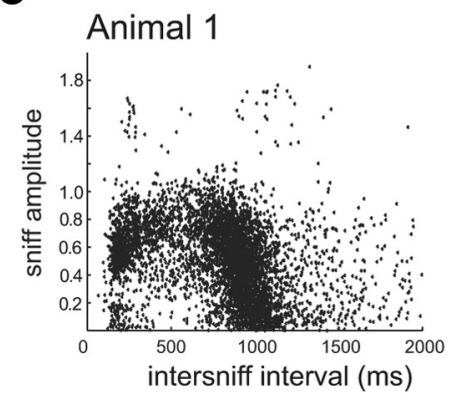

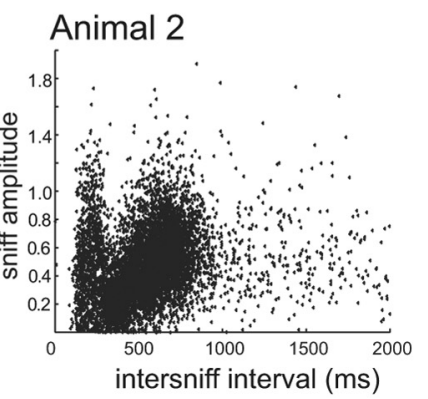

Figure 3. Measuring peak inhalation flow rate in the awake, head-fixed rat. Ai, Aii, Schematic of the lick/no lick, two-odor discrimination (Ai) and passive odorant presentations (Aii) paradigms. For the two-odor discrimination, $S-$ trials were one of two odorants, one of which was strongly sorbed and one weakly sorbed. Imaging data were only collected on $S-$ trials. For the passive paradigm, a tone signaled availability of water reward on all trials, and one of two odorants (strongly or weakly sorbed) was presented in between water presentations. See Materials and Methods for additional details. $\boldsymbol{B}$, Distribution of peak flow rates ("sniff amplitude") for all low-frequency ( $<2.5 \mathrm{~Hz}$ ) inhalations in a behavioral session in two animals. Amplitude scale indicates operational range of flow-rate measurements (see Materials and Methods for details). C, Plots of sniff amplitude versus intersniff interval for the same two animals. Sniff amplitude distributions and amplitude-intersniff interval relationships varied across animals but were consistent across sessions in the same animal (data not shown).

across individual inhalations (Figs. $2 A, 3 B, C$ ). Individual animals showed distinct distributions of sniff amplitudes and distinct relationships between sniff amplitude and frequency (Fig. $3 B, C)$. These distributions differed across animals but were consistent across behavioral sessions repeated on successive days (data not shown). One consistent feature seen in all animals was the expression of a single, large-amplitude inhalation yielding a thermocouple signal at least twice the peak amplitude of other inhalations (Fig. 2A) and repeated approximately once per minute. The appearance of such a signal confirmed that the smalleramplitude, ongoing inhalations did not approach saturation of the thermocouple.

Odorants evoked transient ORN presynaptic calcium signals that were tightly coupled to inhalation, as reported previously (Verhagen et al., 2007; Wesson et al., 2008a). To test whether odorant-evoked ORN responses vary with inhalation flow rate in a manner consistent with the sorption hypothesis in awake rats, we correlated peak inhalation flow with the peak amplitude of the inhalation-coupled calcium transient in individual glomeruli, and for both strongly and weakly sorbed odorants. Inspection of individual trials revealed variability in ORN-response amplitudes that was not apparent during artificial inhalation or sniff playback (Fig. $4 A, B$ ). Inhalations that varied in peak flow rate could evoke similar-amplitude responses, and inhalations with similar peak flow rates could evoke variable-amplitude responses. To quantitatively assess the relationship between peak flow and ORN-response amplitude, we measured the relationship between the thermocouple and optical signal peaks using linear regression, performed separately for each responsive glomerulus in a session. Flow rates were normalized to the operational range of inhalation amplitudes, defined as those covering $94 \%$ of the distribution of all inhalations within a session (excluding the lower and upper $3 \%$ of all inhalations; $n=4147 \pm 1071$ inhalations per session). This operational range varied by a factor of as little as 2 and as much as 15 in different animals (median, 5.8; $n=$ 12 sessions, 5 animals) from the minimal to maximal peak flow rate. Finally, to eliminate confounds of respiration frequency and adaptation on ORN-response amplitudes (Verhagen et al., 2007), we included only the first inhalation after odorant onset and inhalations occurring with an inhalation interval of at least $2 \mathrm{~s}$. Sessions in which first inhalations failed to cover at least $50 \%$ of the operational range were excluded from analysis. We found no difference in inhalation flow rates for the first sniff of odorant in animals performing the active odor discrimination task $(0.49 \pm$ $0.24 ; n=107$ sniffs, 3 animals, 7 sessions) versus those in the passive exposure paradigm $(0.45 \pm 0.18 ; n=118$ sniffs, 2 animals, 5 sessions; $p=0.22$ ), suggesting that rats did not actively modulate sniff strength in this particular context.

Figure $4 C, D$ shows flow-response scatter plots for glomeruli activated by a strongly or a weakly sorbed odorant, normalized to the operational range for that session. For this analysis, strongly sorbed odorants included methyl benzoate $(n=21$ glomeruli, 4 animals) and isovaleric acid ( $n=8$ glomeruli, 3 animals), and weakly sorbed odorants included propyl acetate ( $n=13$ glomeruli, 3 animals) and amyl acetate ( $n=2$ glomeruli, 1 animal). Flow-response slopes, derived from linear fits to peak flow measurements and response amplitudes measured from individual inhalations, were low overall (Fig. $4 C-E$ ). In contrast to the results seen with artificial inhalation and sniff playback, there was no significant difference in the flow-response slopes for weakly versus strongly sorbed odorants: mean slopes for strongly sorbed odorants $(0.10 \pm 0.2, n=29)$ were not significantly higher than for weakly sorbed odorants $(-0.03 \pm 0.19, n=15)(p=0.27$; 2 -sample, unpaired $t$ test). Separately analyzing flow-response slopes for animals performing the active discrimination task or subject to passive odorant presentations also yielded no significant difference between strongly and weakly sorbed odorants or between behavioral paradigm (1-way ANOVA, $F_{(3,40)}=0.76, p=$ 
A

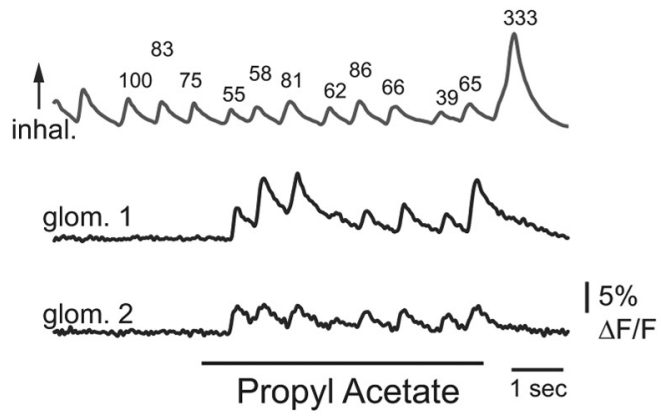

B
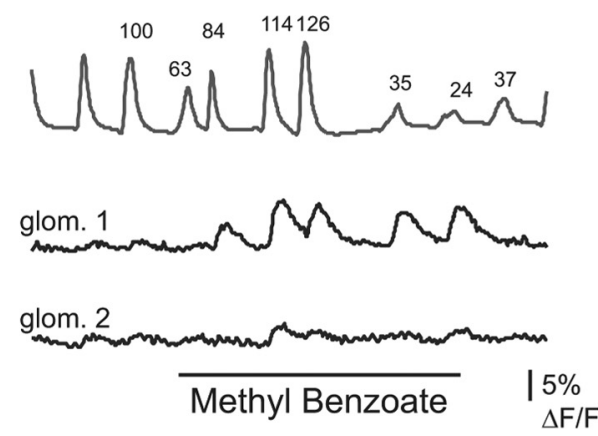

C

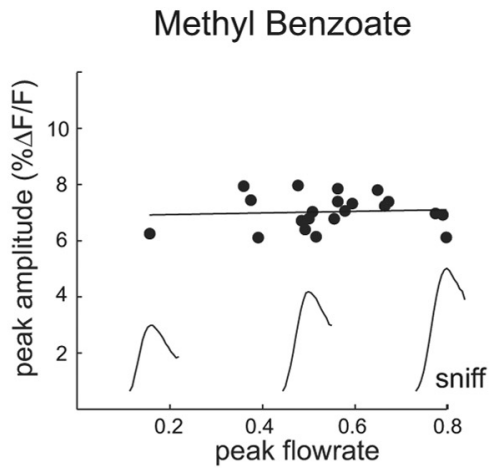

D

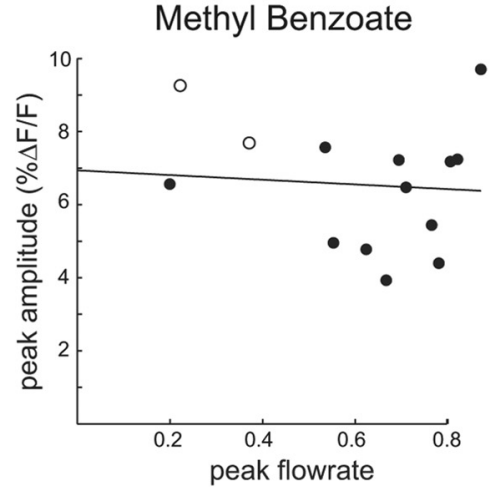

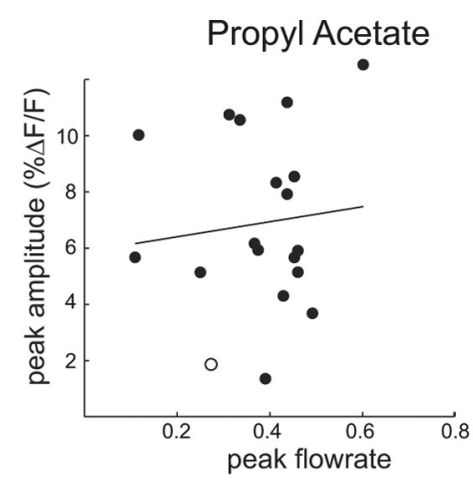

Propyl Acetate

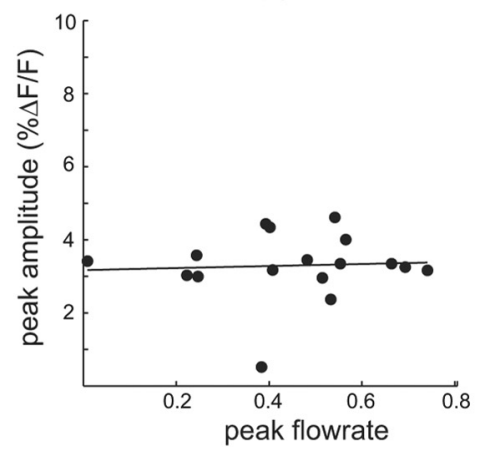

E

$\mathbf{F}$

Slope pairs

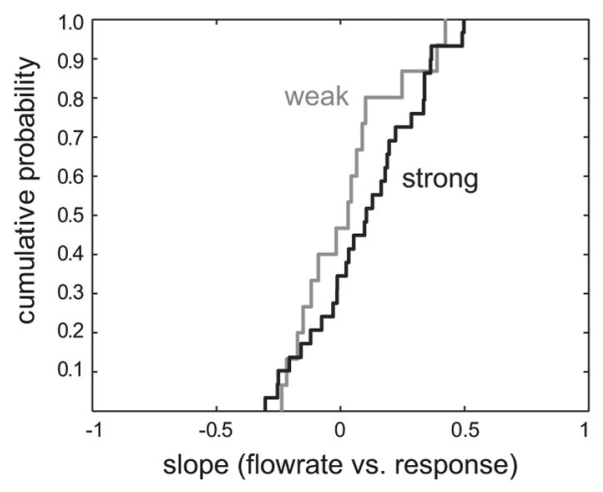

Figure 4. Simultaneous measurement of inhalation flow and ORN-response amplitude in the awake rat. $\boldsymbol{A}, \boldsymbol{B}$, Intranasal airflow measurements (top trace) and presynaptic calcium signals from two ROls measured simultaneously in awake, head-fixed rats for weakly sorbed propyl acetate $(\boldsymbol{A})$ and strongly sorbed methyl benzoate $(\boldsymbol{B})$. Numbers indicate amplitude of the thermocouple signal normalized to that of the first inhalation measured. $A$ is from Animal 2 in Figure $3 B$ (passive odorant presentation); $\boldsymbol{B}$ is from Animal 1 (2-odor discrimination). Note little correlation between relative inhalation amplitudes and ORN responses, especially for propyl acetate. $\boldsymbol{C}, \boldsymbol{D}$, Plots of ORN response versus peak flow rate for the first inhalation in all trials of one session for the animals in $\boldsymbol{A}$ and $\boldsymbol{B}$, respectively. Plots are shown for both propyl acetate and methyl benzoate in each animal. Open circles indicate later inhalations occurring at $>2 \mathrm{~s}$ intersniff interval. Plot in $\boldsymbol{C}$ also shows the thermocouple signal waveform for three inhalations of varying amplitude. Inhalation amplitudes are normalized to the operational range for that session (see Materials and Methods). Line shows linear fit to data. $\boldsymbol{E}$, Cumulative probability distributions for flow rate-response slopes for the first inhalation of odorant in awake, head-fixed rats sampling weakly and strongly sorbed odorants. $x$-Axis is centered around zero slope. Flow rate-response slopes for strongly sorbed odorants are slightly but significantly higher than for weakly sorbed odorants (see Results for details). $\boldsymbol{F}$, Flow-response slope pairs calculated for glomeruli activated by both a weakly and a strongly sorbed odorant in the same preparation.

0.52). Ten glomeruli (in 3 animals) responded to both tested odorants in the same session (in all cases, methyl benzoate and propyl acetate), allowing us to compare flow-response slopes for the same set of ORNs for a weakly and a strongly sorbed odorant (Fig. $4 F$ ). This subset also showed no significant difference in flow-response relationships ( $p=0.3$, paired $t$ test).

Even for the strongly sorbed methyl benzoate and isovaleric acid, which are hypothesized based on their sorption properties to show the strongest dependence on sniff strength (Mozell and Jagodowicz, 1973; Mozell et al., 1991; Oka et al., 2009), the relationship between peak inhalation flow and response amplitude was weak: the mean slope of 0.1 implies that a change in peak flow over the entire range of inhalation flows generated during behavior leads to, on average, a $10 \%$ increase in response amplitude relative to the maximum response measured for that glomerulus. Normalizing response amplitudes to the maximum response measured in any glomerulus for that session (which is an estimate of the dynamic range of responses across all glomeruli) leads to a mean slope value of 0.057 , or $\sim 6 \%$ of the dynamic range of response amplitudes. Flow-response slopes were below 0.2 for $50 \%$ of all glomeruli responding to methyl benzoate and isovaleric acid, implying that even for strongly sorbed odorants 
A

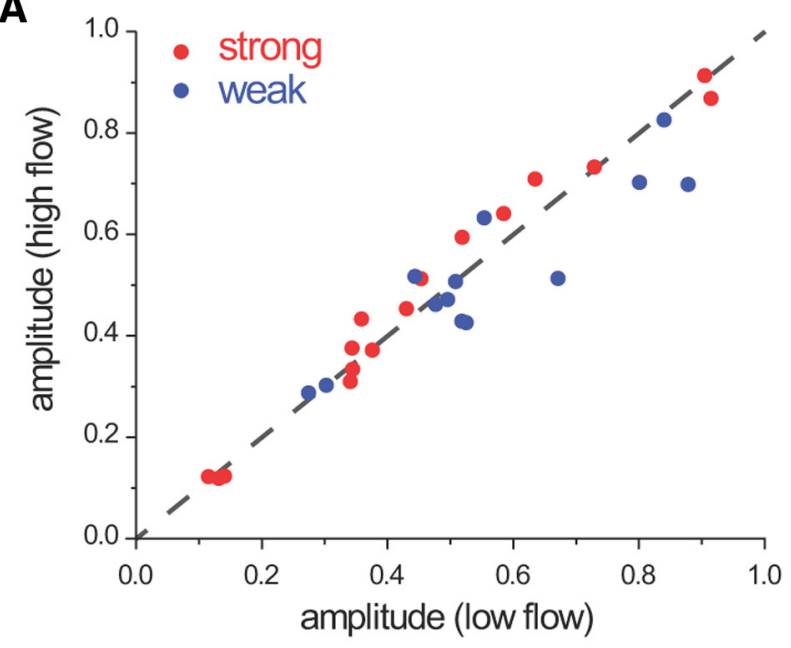

B

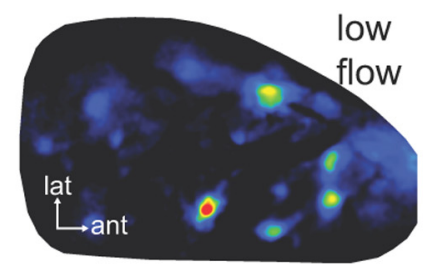

\section{Methyl benzoate}
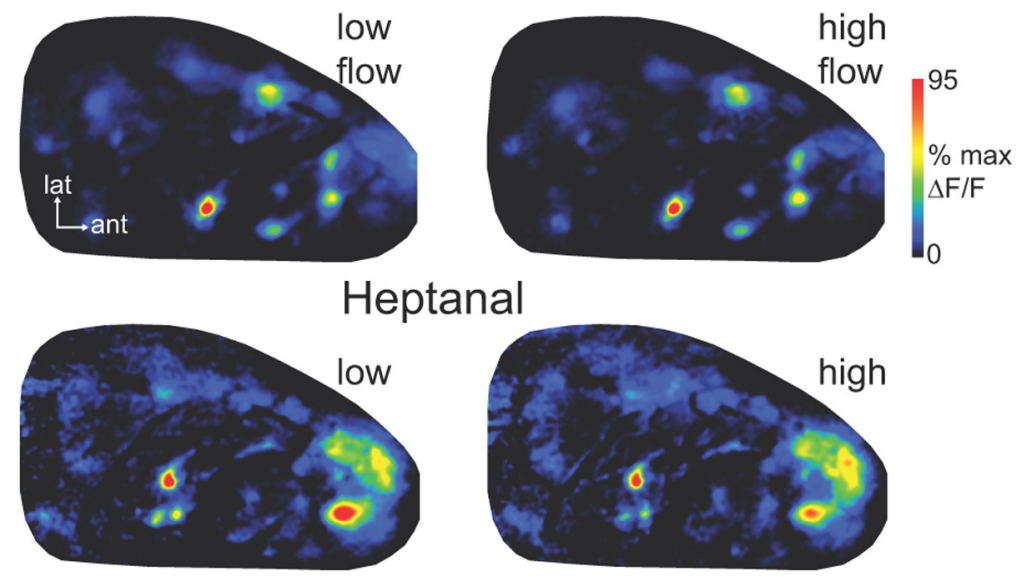

nal

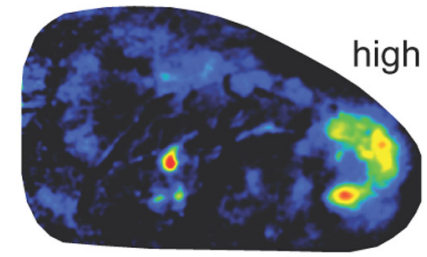

C

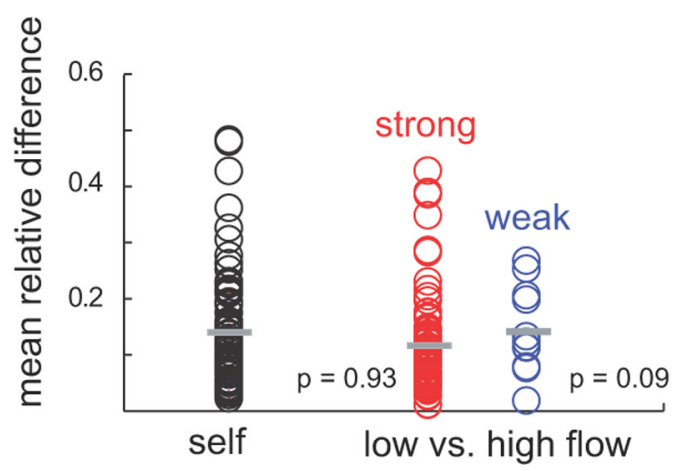

Figure 5. Negligible effect of flow rate on ORN responses during natural odor sampling in the awake rat. $A$, Response amplitudes for ORN inputs to glomeruli (ROls) evoked by low-flow and high-flow inhalations of weakly and strongly sorbed odorants, compiled across five sessions in three animals. Responses are normalized to the maximum response evoked in any glomerulus in that session. $\boldsymbol{B}$, Example of "sniff-triggered average" ORN-response maps for all low-flow and high-flow inhalations in one session, calculated for strongly sorbed methyl benzoate and weakly sorbed heptanal. Each map is normalized to its own maximum. Maps are nearly identical for low-flow and high-flow inhalations. C, Mean difference between response maps evoked by low-flow and high-flow inhalations, calculated from "sniff-triggered average" maps.

sniff strength is, at best, a weak determinant of overall response magnitude.

An additional feature of flow-response relationships measured in the awake rat was the high variance in response amplitudes independent of flow rate. For example, for glomerulus- odor pairs in which the flow-response regression slopes were between -0.1 and 0.1 (implying little effect of flow rate on response amplitudes), the coefficient of variation across individual sniffs was $0.22 \pm 0.07(\sim 22 \%$ of the mean response amplitude). Likewise, for the $25 \%$ of glomerulus-odor pairs with the largest or smallest flow-response slopes $(>0.26$ or $<-0.26)$, the coefficient of determination $\left(r^{2}\right)$ was $0.07 \pm 0.09(n=11)$, implying that flow rate-response relationships explain only a small fraction $(<10 \%)$ of the variance in $\mathrm{ORN}$ responses.

To further test for effects of inhalation flow rate using a measure that did not depend on a particular quantitative relationship between flow rate and response amplitude, we looked at a subset of sessions with a sufficient number of trials in which we divided inhalations into highflow and low-flow groups (defined as the first and fourth quartile of the dynamic range of flow rates for a session) and compared ORN responses between these groups (Fig. 5A). We found no significant difference in response amplitude for highflow versus low-flow sniffs for either strongly or weakly sorbed odorants (paired $t$ test, $n=$ 16 strongly sorbed, $n=13$ weakly sorbed pairs; $p>0.1$ for both groups).

Finally, we asked whether high-flow or low-flow sniffs generate different patterns of ORN input across glomeruli of the dorsal $\mathrm{OB}$, a result expected if changes in flow rate differentially recruit input to different glomeruli. This analysis tested the more general hypothesis that sniff strength can modulate primary odor representations by altering patterns of glomerular activation, as occurs using artificial inhalation (Oka et al., 2009; Figs. 1B, 2C). "Snifftriggered average" response maps, generated for odorants sampled with high-flow or low-flow sniffs, appeared nearly identical for both strongly and weakly sorbed odorants (Fig. 5B). We quantitatively compared response maps using the $\mathrm{MD}$ across all activated glomeruli in a map (Fig. 5C) (Verhagen et al., 2007). MD values between high-flow and low-flow sniffs of either strongly or weakly sorbed odorants were not significantly different from those of repeated sniffs of the same odorant $\left(\mathrm{MD}_{\text {self }}\right.$, $0.14 \pm 0.1, n=85$ map comparisons; $\mathrm{MD}_{\text {hilow }}$ for strongly sorbed odorants, $0.11 \pm 0.08, n=86$ map comparisons, $p=$ 0.18 ; $\mathrm{MD}_{\text {hilow }}$ for weakly sorbed odorants, $0.14 \pm 0.08, n=12$ map comparisons, $p=0.53$; two-sided Wilcoxon rank sum test). Overall these results suggest that increases in inhalation flow rate do not significantly alter odorant representations over the range of flow rates generated during natural odor sampling in the awake animal. 
A

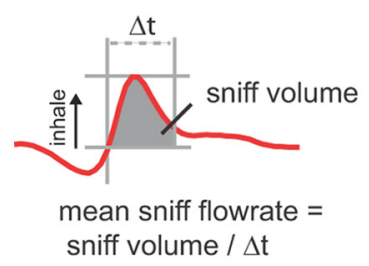

B

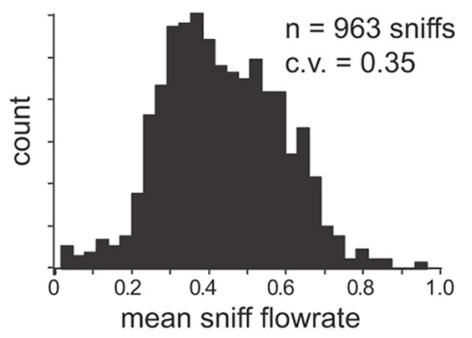

C

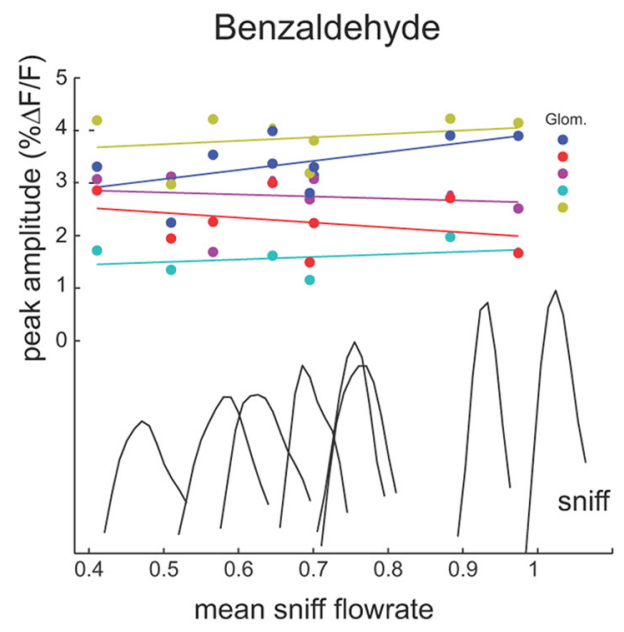

D

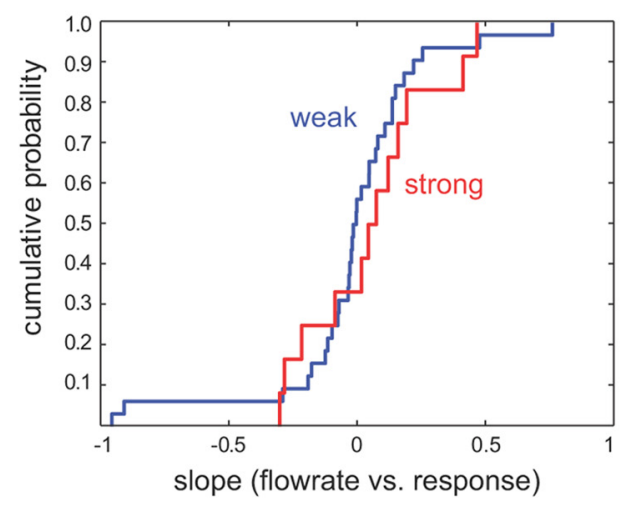

E

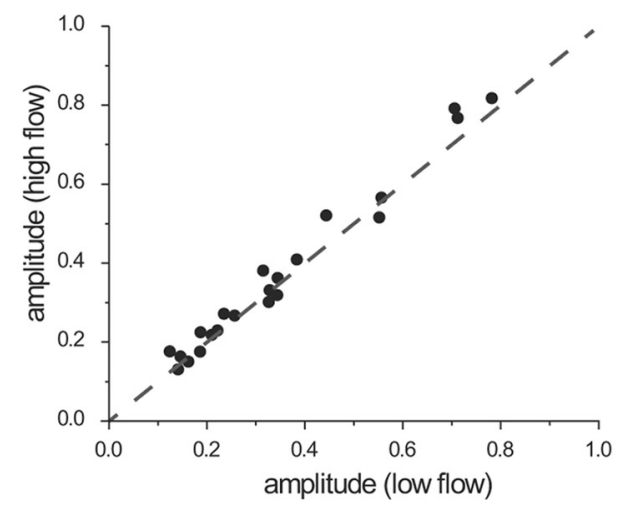

Figure 6. ORN-response amplitudes are not shaped by mean inhalation flow rate during natural odor sampling. $A$, Schematic illustrating measurement of mean inhalation flow rate from intranasal pressure recordings in awake, head-fixed rats. Mean flow rate is estimated from inhalation volume (gray-shaded area) divided by inhalation duration $(\Delta t)$. $B$, Histogram of mean flow rates measured in one behavioral session and scaled as for peak flow measurements. C, Plots of ORN response versus mean inhalation flow rate for the first inhalation in all trials of
To strengthen this conclusion, we used a different measure of inhalation flow rate to repeat this experiment in a different set of animals. Odorant sampling involves dynamic changes in airflow driven by pressure transients generated by the respiratory system, and odorant uptake by the epithelium could be driven by flow over the course of inhalation rather than the peak flows reported by thermocouple measurement. Thus, in a separate set of experiments, we tested for correlations between ORN-response amplitudes and the mean flow generated across the inhalation phase of respiration, using intranasal pressure measurements in headfixed rats (Verhagen et al., 2007). Mean flow rate was estimated from the integral of the inspiration pressure measured from the dorsal recess, divided by the duration of the inspiratory phase (see Materials and Methods) (Fig. 6A). Inspiration duration was $122 \pm 54 \mathrm{~ms}$ ( $n=16,226$ sniffs, 8 sessions in 5 rats). Mean flow measurements were then normalized into an operational range as was done for the thermocouple signals. The variance of mean flow rates throughout a session was similar to that of the thermocouple signals (Fig. 6B).

As with the peak flow measurements, we observed little relationship between mean flow and response amplitudes. Mean inhalation flow and response amplitudes were correlated for first inhalations of odorant as described for peak flow measurement (Fig. 6C). Flow-response slopes for the strongly sorbed benzaldehyde (measured from 12 glomeruli in 1 animal) were low (median, 0.05; Fig. $6 D$ ) and were not significantly higher than for slope values measured for two other weakly or moderately sorbed odorants (amyl acetate, 3 glomeruli in 2 animals; methyl valerate, 21 glomeruli in 4 animals; median $=-0.01$; Kolmogorov-Smirnov test, $p=0.527)$. Comparing average response amplitudes for all low-flow versus high-flow sniffs of odorant in a subset of the data ( $n=22$ glomerulus-odor pairs, 3 animals) (Fig. $6 E$ ), as done for the thermocouple measurements, did reveal a significant difference in response amplitude for strongly sorbed benzaldehyde (paired Wilcoxon signed rank test, $p=0.01$ ) but not for methyl valerate ( $p=0.15$ ). While significant for benzaldehyde, the magnitude of this difference was small (median, $1.8 \%$ increase in amplitude as a fraction of the response amplitude dynamic range). Thus, using two different measures of inhalation flow during natural odor sampling, we found little evidence that odorant sorption properties affect the relationship between sniff strength and ORN-response magnitude, and that sniff strength, even for strongly sorbed odorants, does not substantially alter the magnitude or spatial patterns of glomerular input thought to represent odor identity.

\section{Testing for behavioral control of sniff flow rate in relation to odorant sorption}

A final prediction of the sorption hypothesis is that animals are capable of altering inhalation flow rates in a way that optimizes their ability to detect or discriminate odorants (Youngentob et al., 1987; Kent et al., 1996; Schoenfeld and Cleland, 2005; Mainland and Sobel, 2006). Thus, we designed a behavioral paradigm to ask whether rats alter sniff strength in a manner consistent with

$\leftarrow$

strongly sorbed benzaldehyde in one session. Responses are plotted for five glomeruli and normalized as in Figure 4C. Traces show the intranasal pressure waveform for each inhalation in the plot. Lines show linear fit to each data series. $\boldsymbol{D}$, Cumulative probability distributions for mean flow rate-response slopes for first inhalations in awake, head-fixed rats sampling strongly sorbed (red) and other odorants (blue), normalized as in Figure 5A. E, Normalized response amplitudes for $\mathrm{ORN}$ inputs to glomeruli evoked by low-flow and high-flow inhalations of all odorants. 
this prediction. We measured peak inhalation flow using a thermocouple in head-fixed rats performing a lick/no-lick two-odor discrimination task, as described above. In this variant of the paradigm, five rats were trained to discriminate between two odorants with similar sorption properties: either two weakly or two strongly sorbed odorants (Table 1). The strongly sorbed pair was methyl benzoate-benzaldehyde in all cases. The weakly sorbed pair were propyl acetate-butyl acetate for two animals and propyl acetate-amyl acetate for three animals. During training, odorant concentration was set to $\sim 4 \mathrm{ppm}$ for all odorants (see Materials and Methods), which is well below levels that elicit trigeminal responses in rats or lead to irritation or sniff suppression in humans (Silver and Moulton, 1982; Johnson et al., 2003). After achieving performance criterion, the concentration of both odorants was decreased in successive blocks of 50 trials until performance dropped to near-chance levels (below 60\% correct for two successive blocks). The same animals were subsequently retrained to discriminate the second odorant pair (both weakly or strongly sorbed), and the series of decreasing concentrations repeated.

The rationale for using pairs of odorants with similar sorption properties was that this design allows the rat to adopt a strategy that is most effective at discriminating (or detecting, since the underlying perceptual process is not clear in a 2-choice task) odorants with "known" relationships to flow rate by familiarizing the rat with the odorants in training sessions and by ensuring that this relationship is similar for both odorants. Presenting odorant pairs with different sorption properties would not be predicted to lead to sorption-related changes in sampling strategy, as odorant identity (and, thus, sorption) would be unknown until after discrimination had occurred. Using our chosen paradigm, we predicted that, if increased flow rate leads to increased odorant uptake for strongly but not weakly sorbed odorants as predicted by the sorption hypothesis, rats would show an increase in sniff strength (i.e., peak inhalation flow) as odorant concentration decreased to levels near their detection threshold for strongly sorbed odorants, but little or no change in sniff strength for weakly sorbed odorants. Figure $7 A$ shows an example of peak inhalation flows measured over the course of an odorant presentation, averaged across all 50 trials in each block. Rats showed significant modulation of sniff strength immediately after odorant presentation (1-way ANOVA, $F_{(1,42)}=85.7, p$ value $\ll 0.001$ ). Modulation was most commonly manifest as a decrease in peak flow rate relative to preodor inhalations (e.g., Fig. $7 A, B$ ), although the degree and time course of modulation varied across individual rats (compare Fig. $7 A-C$ ). This result is consistent with our previous reports of a decrease in both sniff strength (peak inhalation pressure) and frequency during the sampling of familiar odorants (Verhagen et al., 2007; Wesson et al., 2009).

We next asked whether rats modulated sniff strength as a function of odorant concentration, and whether they did so differently for strongly versus weakly sorbed odorants. Inspection of peak inhalation flows tracked across successively lower odorant concentrations showed no clear change in sniff strength for either strongly or weakly sorbed odorants, even as animals reached discrimination threshold (Fig. $7 B$ ). These results were confirmed statistically, with no significant effect of either odorant sorption or concentration on peak inhalation flow rate for sniffs taken after odorant presentation but before licking (2-way ANOVA; odorant sorption factor: $F_{(1,13)}=1.02, p=0.33$; dilution factor: $\left.F_{(4,13)}=1.34, p=0.31\right)$, and no significant interaction between sorption and concentration (sorption-dilution interaction: $\left.F_{(3,13)}=0.095, p=0.96\right)$. Because this paradigm involved a large number of trials at each concentration to ensure high performance levels (100 trials across 2 sessions), we also tested for sorption-related differences in sniff strength in two additional animals in which odorant concentration was decreased in four successive blocks of 25 trials within a single session (Fig. 7C,D). These two rats showed no modulation of peak inhalation flow by odorant presentation (1-way ANOVA: $F_{(1,22)}=0.59, p=0.45$ ), no modulation as a function of odorant concentration, and no interaction between concentration and sorption (2-way ANOVA, odorant sorption: $F_{(1,6)}=1.72, p=0.24$; dilution: $F_{(3,6)}=0.46$, $p=0.72$; sorption-dilution interaction: $F_{(1,6)}=0.13, p=0.73$ ). Overall these results indicate that, while rats can actively modulate sniff strength in the context of odor discrimination, they do not differentially modulate sniff strength for odorants with different sorption properties, nor do they appear to increase sniff strength to enhance their ability to discriminate odorants of diminishing concentration.

\section{Discussion}

Differential deposition of odorant across the olfactory epithelium was among the earliest hypothesized mechanisms for generating odorant-specific response patterns across ORNs (Adrian, 1950; Mozell, 1964), and its dependence on both physicochemical properties and intranasal airflow has long been proposed to enable active control of odor representations by changing the rate and volume of inhaled odorant during sniffing (Hornung et al., 1987a,b; Mozell et al., 1987; Youngentob et al., 1987; Schoenfeld and Cleland, 2005). This study tests these ideas in awake, behaving animals; we tested predictions from earlier work using in vivo imaging of ORN responses in anesthetized and awake rats as well as a behavioral test for active modulation of inhalation flow during odor discrimination. While we confirmed that artificial changes in flow rate can alter ORN responses consistent with predictions of the sorption hypothesis (Hahn et al., 1994; Keyhani et al., 1997; Oka et al., 2009), we also found that sniff strength has negligible effect on responses during natural sniffing and that behaving rats do not modulate flow rate to improve odorant discrimination. Overall these results suggest that, contrary to longstanding predictions, sniff strength does not shape odor representations sufficiently to be part of a strategy for active odor sensing in the behaving animal. Instead, maintaining airflow-invariant odor representations may be a more adaptive mechanism for encoding odor information during behavior.

Optical imaging was restricted to the dorsal OB, and we cannot exclude the possibility that flow-rate effects might emerge for ORNs projecting to other bulbar regions. ORNs projecting to the dorsal $\mathrm{OB}$ are mainly located in the central region of the epithelium, which is earliest in the inhaled flow path, while ORNs projecting to lateral and ventral $\mathrm{OB}$ are located in the peripheral epithelium, which is later in the flow path and where flow rates are slower (Schoenfeld and Cleland, 2006; Yang et al., 2007a). Thus, flow-dependent effects might be more significant for the lateral and ventral OB. Nonetheless, the dorsal OB is well suited for testing the strongest prediction from the sorption hypothesis (i.e., that strongly sorbed odorants should show a strong dependence on flow rate). Many ligands for dorsal-projecting ORNs are strongly sorbed (Bozza and Kauer, 1998; Bozza et al., 2009), and we measured airflow directly from around the region of the epithelium that projects to the dorsal $\mathrm{OB}$. In addition, the fact that flow-response relationships consistent with the sorption hypothesis were apparent during artificial inhalation suggests that such relationships should have been apparent on the dorsal OB had they persisted during natural sampling. 

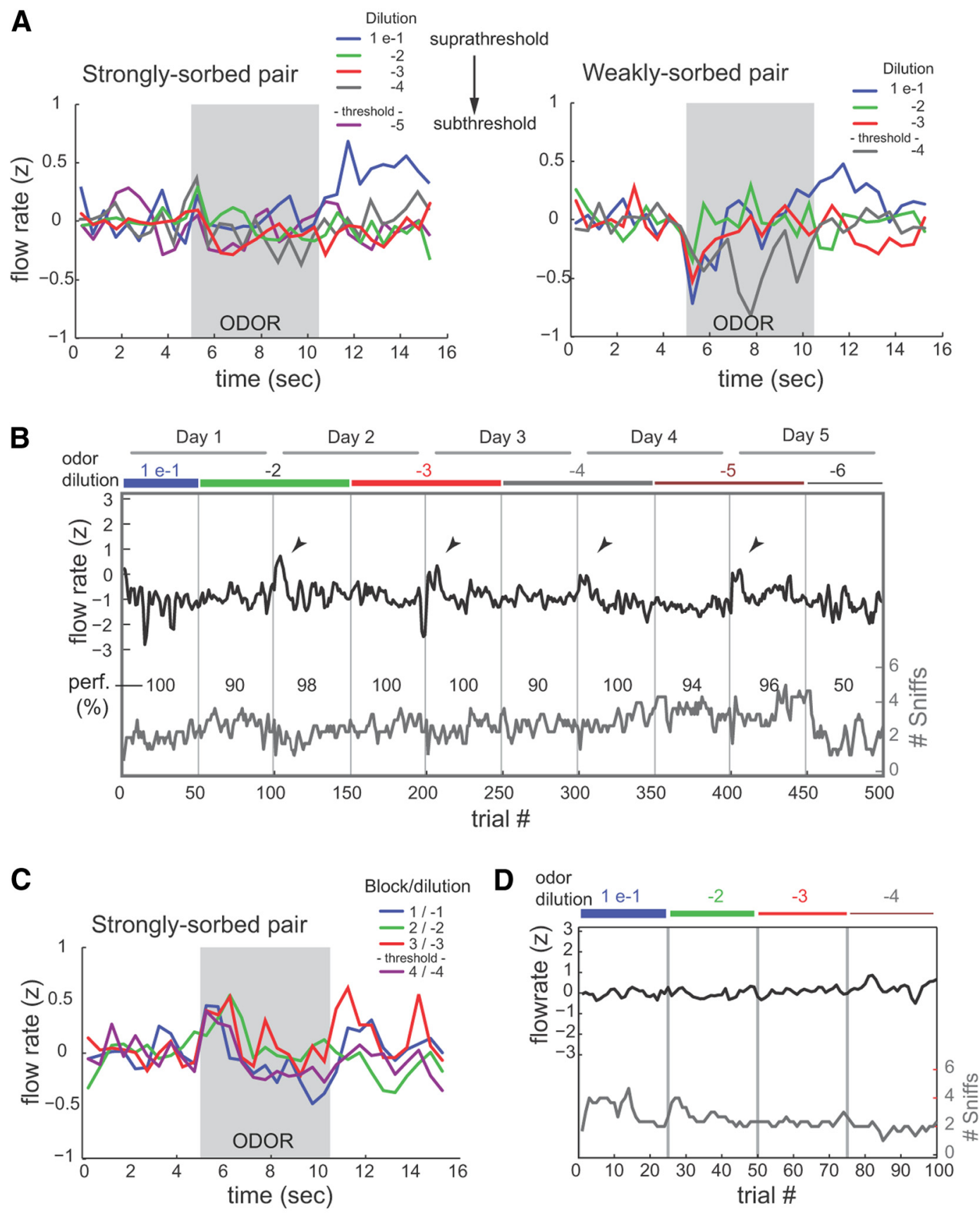

Figure 7. Head-fixed rats do not modulate sniff strength when performing a two-odor discrimination task. A, Plots of peak inhalation flow rate versus time relative to odor presentation for a rat performing lick/no-lick discriminations of a pair of strongly sorbed (methyl benzoate- benzaldehyde) or weakly sorbed (propyl acetate-amyl acetate) odorants. Peak flow rates are $z$-scored within each session, binned in $500 \mathrm{~ms}$ bins, and averaged across all trials within a block (100 trials). This rat shows a modest suppression in flow rate during odor presentation but no change as odorant concentrations decrease to below performance threshold. $\boldsymbol{B}$, Peak flow rates for inhalations of odorant made before licking, plotted for all trials in one dilution series for the strongly sorbed pair methyl benzoate- benzaldehyde; different rat than $A$. Dilution blocks (blue) and session days are indicated above plot and demarcated by vertical lines. Red plot shows number of sniffs after odorant presentation and before mean lick time (median, 3). Numbers (perf) indicate performance per block, in percentage correct trials. Plots are smoothed with a 3 bin moving average for display. Note increase in flow rate for a few trials at the start of each day (arrowheads), but no change as concentration was decreased. $C$, $\boldsymbol{D}$, Peak inhalation flow rate versus time ( $\boldsymbol{C}$ ) and flow versus trial (D) for a different rat in which odorant pair concentrations were decreased in successive blocks within one session (100 total trials, 25 trials per block).

\section{Biophysical constraints on inhalation flow rate as a modulator of odor representations}

Constraints imposed by the biophysics of odorant-ORN interactions may explain why modulating sniff strength does not lead to reliable changes in ORN responses during natural sniffing. First, compared with artificial inhalation, natural sniffs generate more gradual changes in flow rate, meaning that flow-rate differences generated by sniffs of different amplitude will emerge relatively gradually, reaching a peak $40 \mathrm{~ms}$ or more after inhalation begins (Roux et al., 2006; Hegoburu et al., 2011). Second, ORNs preferentially respond to odorant onset and have short integration times before spike-rate adaptation limits responsiveness (Duchamp-Viret et al., 1999; Ghatpande and Reisert, 2011). Thus flow-rate differences that emerge slowly may have little opportunity to modulate ORN output. This effect may explain why sorption-related effects were weaker for "playback" of natural 
sniffs than for the artificial inhalation pulses. Third, flow ratesorption relationships are nonlinear and fit predictions of the sorption hypothesis only over a limited range, and the range of flow rates causing the greatest modulation of sorption differs for different odorants (Hahn et al., 1994). Fourth, because of the nonlinear concentration dependence of ORNs (Firestein et al., 1993), changes in the amount of odorant absorbed onto the epithelium may lead to substantial changes in ORN spike output only over a limited range of concentrations. Together, these constraints leave a small parameter space in which changes in inhaled flow rate can significantly shape patterns of activity across ORN populations in a manner reliable enough to be used in odor detection or discrimination.

Another important constraint on a flow rate-based sampling strategy is the variability in ORN responses from sniff to sniff. For example, changes in peak inhalation flow rate could, even for strongly sorbed odorants, account for $<10 \%$ of the variance in ORN responses after eliminating the confound of inhalation frequency. The higher variability in awake versus anesthetized animals likely reflects real variance in the strength of ORN activation as opposed to technical factors related to the imaging approach, as response variation diminishes in head-fixed animals acutely subject to anesthesia (data not shown, but see Verhagen et al., 2007). This variance may be due to variance in airflow patterns from sniff to sniff driven by other parameters of respiration, positioning of the external nares, or modulation of calcium influx at the presynaptic terminal (Wachowiak et al., 2005). Regardless of its source, the variance in ORN responses due to factors other than flow rate also limits the use of flow-rate modulation as part of an active strategy for sampling olfactory information.

\section{Active sensing strategies and odor coding}

In behavioral experiments, head-fixed rats did not modulate sniff strength as the intensity of two odorants decreased to below threshold for discrimination, even for strongly sorbed odorants whose uptake by the olfactory epithelium is predicted to have the greatest dependence on flow rate (Mozell et al., 1987; Hahn et al., 1994). We previously observed a similar lack of concentrationdependent sniff-strength modulation using pressure measurements in head-fixed rats discriminating two odorants with divergent sorption properties (Wesson et al., 2009). These results are consistent with the conclusion that flow-rate modulation does not shape ORN responses in a manner adaptive for odor detection tasks. We note, however, that Rojas-Libano and Kay (2012) recently reported behavioral evidence that rats modify sniffing parameters, including but not limited to sniff flow rate, as a function of odorant sorption when detecting components of a mixture. Further work will be necessary to resolve the differences between these two studies. Sniff-strength modulation also likely plays a role in other contexts, albeit independent of odorant sorption. For example, in rodents sniff strength can shape the dynamics of postsynaptic activity in the OB (Courtiol et al., 2011b; Esclassan et al., 2012), and can alter the temporal response patterns of individual mitral/tufted cells relative to inhalation (Courtiol et al., 2011a; Shusterman et al., 2011). Rodents and humans also exhibit sniff suppression in response to aversive or irritating concentrations (Johnson et al., 2003) and even during repeated sampling of familiar odors at concentrations that elicit appetitive (i.e., nonaversive) responses (Verhagen et al., 2007; Wesson et al., 2009). Thus, one important role for modulating sniff strength may be to limit unnecessary exposure to odorants, although we did not find evidence that such modulation varies with odorant sorption (Fig. 7A). Increases in sniff strength may also be used to overcome limited access to odorant [e.g., from a still-air reservoir (Youngentob et al., 1987) or from trace amounts on a substrate]. Rats and humans may also modulate other sniff parameters, such as duration, to control odorant access (Sobel et al., 2000). Finally, flow rate-sorption interactions may lead to perceptible differences in odor quality or intensity in humans, where inhalation durations are longer (Sobel et al., 1999). Whether modulating sniff strength aids in odor discrimination during natural sniffing in humans remains to be tested, however.

Despite a limited effect of flow rate during sniffing, there is strong evidence that sorption plays a fundamental role in olfaction by shaping how odorants are differentially distributed across the olfactory epithelium as they pass through the nasal cavity. Chromatographic separation of odorants with different sorption properties has been measured experimentally (Mozell, 1964; Mozell and Jagodowicz, 1973) and mapped directly using voltagesensitive dye imaging (Kent et al., 1996) and electro-olfactogram recordings (Scott et al., 2000). Recent evidence suggests that patterns of odorant-receptor expression across the epithelium match patterns of odorant sorption (Schoenfeld and Cleland, 2005; Scott, 2006). Our results are consistent with this model, but suggest that the variations in intranasal flow rate that occur during natural odor sampling do not significantly alter ORNresponse patterns. Indeed, it is possible that olfactory receptor distributions are organized to minimize the effects of changes in flow rate on ORN-response patterns to maintain an invariance in odor representations in the face of changing inhalation patterns caused by the varying respiratory or metabolic demands that occur during behavior. Additional fine-scale modeling of odorant sorption patterns during natural sniff waveforms combined with mapping of expression patterns for olfactory receptors with known ligands will be important to test this idea.

Aside from flow-rate modulation, rodents and other species likely employ other olfactory sampling strategies in other behavioral contexts. One sampling parameter that does appear to robustly shape early odorant representations is sniff frequency. Rats (and mice) modulate respiration frequency dramatically, from $1-2 \mathrm{~Hz}$ at rest to $6-12 \mathrm{~Hz}$ during active sniffing, and display temporally precise and context-dependent changes in frequency during odor-guided behaviors (Welker, 1964; Kepecs et al., 2007; Wesson et al., 2008b, 2009). Using the same approach as in the present study, we have found that high-frequency sniffing leads to significant attenuation of ORN-response magnitudes, which can reduce the salience of background odorants during olfactory search behavior (Verhagen et al., 2007). It would be interesting to explore whether frequency-dependent attenuation differs for odorants with different sorption properties due to differences in the dynamics of odorant uptake and clearance. In general, examining how different active sensing strategies shape how sensory information processing remains a rich avenue for future studies combining neural recordings with behavioral approaches.

\section{References}

Adrian ED (1950) Sensory discrimination with some recent evidence from the olfactory organ. Br Med Bull 6:330-333. Medline

Amoore J, Buttery R (1978) Partition coefficients and comparative olfactometry. Chem Senses and Flavour 3:57-71. CrossRef

Bozza TC, Kauer JS (1998) Odorant response properties of convergent olfactory receptor neurons. J Neurosci 18:4560-4569. Medline

Bozza T, Vassalli A, Fuss S, Zhang JJ, Weiland B, Pacifico R, Feinstein P, Mombaerts P (2009) Mapping of class I and class II odorant receptors to 
glomerular domains by two distinct types of olfactory sensory neurons in the mouse. Neuron 61:220-233. CrossRef Medline

Carey RM, Wachowiak M (2011) Effect of sniffing on the temporal structure of mitral/tufted cell output from the olfactory bulb. J Neurosci 31: 10615-10626. CrossRef Medline

Carey RM, Verhagen JV, Wesson DW, Pírez N, Wachowiak M (2009) Temporal Structure of Receptor Neuron Input to the Olfactory Bulb Imaged in Behaving Rats. J Neurophysiol 101:1073-1088. Medline

Cheung MC, Carey RM, Wachowiak M (2009) A method for generating natural and user-defined sniffing patterns in anesthetized or reduced preparations. Chem Senses 34:63-76. Medline

Courtiol E, Amat C, Thevenet M, Messaoudi B, Garcia S, Buonviso N (2011a) Reshaping of bulbar odor response by nasal flow rate in the rat. PLoS One 6:e16445. CrossRef Medline

Courtiol E, Hegoburu C, Litaudon P, Garcia S, Fourcaud-Trocme N, Buonviso N (2011b) Individual and synergistic effects of sniffing frequency and flow rate on olfactory bulb activity. J Neurophysiol 106:2813-2824. CrossRef Medline

Dethier VG (1987) Sniff, flick, and pulse: an appreciation of interruption. Proc Am Philosophical Soc 131:159-176.

Duchamp-Viret P, Chaput MA, Duchamp A (1999) Odor response properties of rat olfactory receptor neurons. Science 284:2171-2174. CrossRef Medline

Esclassan F, Courtiol E, Thévenet M, Garcia S, Buonviso N, Litaudon P (2012) Faster, deeper, better: the impact of sniffing modulation on bulbar olfactory processing. PLoS ONE 7:e40927. CrossRef Medline

Firestein S, Picco C, Menini A (1993) The relation between stimulus and response in olfactory receptor cells of the tiger salamander. J Physiol 468:1-10. Medline

Ghatpande AS, Reisert J (2011) Olfactory receptor neuron responses coding for rapid odor sampling. J Physiol 589:2261-2273. CrossRef Medline

Hahn I, Scherer PW, Mozell MM (1994) A mass transport model of olfaction. J Theor Biol 167:115-128. CrossRef Medline

Hegoburu C, Shionoya K, Garcia S, Messaoudi B, Thevenet M, Mouly A-M (2011) The RUB cage: respiration-ultrasonic vocalizations-behavior acquisition set-up for assessing emotional memory in rats. Front Behav Neurosci 5:25. Medline

Hornung D, Mozell M (1981) Accessibility of odorant molecules to the receptors. In: Biochemistry of taste and olfaction (Cagan R, Kare M, eds), pp 33-45. New York: Academic.

Hornung DE, Leopold DA, Youngentob SL, Sheehe PR, Gagne GM, Thomas FD, Mozell MM (1987a) Airflow patterns in a human nasal model. Arch Otolaryngol Head Neck Surg 113:169-172. CrossRef Medline

Hornung DE, Youngentob SL, Mozell MM (1987b) Olfactory mucosa/air partitioning of odorants. Brain Res 413:147-154. CrossRef Medline

Johnson BN, Mainland JD, Sobel N (2003) Rapid olfactory processing implicates subcortical control of an olfactomotor system. J Neurophysiol 90:1084-1094. CrossRef Medline

Kent PF, Mozell MM, Murphy SJ, Hornung DE (1996) The interaction of imposed and inherent olfactory mucosal activity patterns and their composite representation in a mammalian species using voltage-sensitive dyes. J Neurosci 16:345-353. Medline

Kepecs A, Uchida N, Mainen ZF (2007) Rapid and precise control of sniffing during olfactory discrimination in rats. J Neurophysiol 98:205-213. CrossRef Medline

Keyhani K, Scherer PW, Mozell MM (1997) A numerical model of nasal odorant transport for the analysis of human olfaction. J Theor Biol 186: 279-301. CrossRef Medline

Kurtz DB, Zhao K, Hornung DE, Scherer P (2004) Experimental and numerical determination of odorant solubility in nasal and olfactory mucosa. Chem Senses 29:763-773. CrossRef Medline

Mainland J, Sobel N (2006) The sniff is part of the olfactory percept. Chem Senses 31:181-196. Medline

Malnic B, Hirono J, Sato T, Buck LB (1999) Combinatorial receptor codes for odors. Cell 96:713-723. CrossRef Medline

Mozell MM (1964) Evidence for sorption as a mechanism of the olfactory analysis of vapours. Nature 203:1181-1182. CrossRef Medline

Mozell MM (1970) Evidence for a chromatographic model of olfaction. J Gen Physiol 56:46-63. CrossRef Medline

Mozell MM, Jagodowicz M (1973) Chromatographic separation of odorants by the nose: retention times measured across in vivo olfactory mucosa. Science 181:1247-1249. CrossRef Medline
Mozell MM, Sheehe PR, Hornung DE, Kent PF, Youngentob SL, Murphy SJ (1987) Imposed and inherent mucosal activity patterns. Their composite representation of olfactory stimuli. J Gen Physiol 90:625-650. CrossRef Medline

Mozell MM, Kent PF, Murphy SJ (1991) The effect of flow-rate upon the magnitude of the olfactory response differs for different odorants. Chem Senses 16:631-649. CrossRef

Oka Y, Takai Y, Touhara K (2009) Nasal airflow rate affects the sensitivity and pattern of glomerular odorant responses in the mouse olfactory bulb. J Neurosci 29:12070-12078. CrossRef Medline

Rojas-Libano D, Kay LM (2012) Interplay between sniffing and odorant sorptive properties in the rat. J Neurosci 32:15577-15589. CrossRef

Roux SG, Garcia S, Bertrand B, Cenier T, Vigouroux M, Buonviso N, Litaudon P (2006) Respiratory cycle as time basis: an improved method for averaging olfactory neural events. J Neurosci Methods 152:173-178. CrossRef Medline

Schoenfeld TA, Cleland TA (2005) The anatomical logic of smell. Trends Neurosci 28:620-627. CrossRef Medline

Schoenfeld TA, Cleland TA (2006) Anatomical contributions to odorant sampling and representation in rodents: zoning in on sniffing behavior. Chem Senses 31:131-144. Medline

Scott JW (2006) Sniffing and spatiotemporal coding in olfaction. Chem Senses 31:119-130. Medline

Scott JW, Brierley T, Schmidt FH (2000) Chemical determinants of the rat electro-olfactogram. J Neurosci 20:4721-4731. Medline

Scott JW, Acevedo HP, Sherrill L (2006) Effects of concentration and sniff flow rate on the rat electroolfactogram. Chem Senses 31:581-593. CrossRef Medline

Scott-Johnson PE, Blakley D, Scott JW (2000) Effects of air flow on rat electroolfactogram. Chem Senses 25: 25:761-768. CrossRef

Shusterman R, Smear MC, Koulakov AA, Rinberg D (2011) Precise olfactory responses tile the sniff cycle. Nat Neurosci 14:1039-1044. CrossRef Medline

Silver WL, Moulton DG (1982) Chemosensitivity of rat nasal trigeminal receptors. Physiol Behav 28:927-931. CrossRef Medline

Sobel N, Khan RM, Saltman A, Sullivan EV, Gabrieli JD (1999) The world smells different to each nostril. Nature 402:35. CrossRef Medline

Sobel N, Khan RM, Hartley CA, Sullivan EV, Gabrieli JD (2000) Sniffing longer rather than stronger to maintain olfactory detection threshold. Chem Senses 25:1-8. CrossRef Medline

Spors H, Wachowiak M, Cohen LB, Friedrich RW (2006) Temporal dynamics and latency patterns of receptor neuron input to the olfactory bulb. J Neurosci 26:1247-1259. CrossRef Medline

Uchida N, Kepecs A, Mainen ZF (2006) Seeing at a glance, smelling in a whiff: rapid forms of perceptual decision making. Nat Rev Neurosci 7:485-491. CrossRef Medline

Verhagen JV, Wesson DW, Netoff TI, White JA, Wachowiak M (2007) Sniffing controls an adaptive filter of sensory input to the olfactory bulb. Nat Neurosci 10:631-639. CrossRef Medline

Wachowiak M (2011) All in a sniff: olfaction as a model for active sensing. Neuron 71:962-973. CrossRef Medline

Wachowiak M, Cohen LB (2001) Representation of odorants by receptor neuron input to the mouse olfactory bulb. Neuron 32:723-735. CrossRef Medline

Wachowiak M, McGann JP, Heyward PM, Shao Z, Puche AC, Shipley MT (2005) Inhibition of olfactory receptor neuron input to olfactory bulb glomeruli mediated by suppression of presynaptic calcium influx. J Neurophysiol 94:2700-2712. CrossRef Medline

Welker WI (1964) Analysis of sniffing in the albino rat. Behavior 22:223244. CrossRef

Wesson DW, Carey RM, Verhagen JV, Wachowiak M (2008a) Rapid encoding and perception of novel odors in the rat. PLoS Biology 6:e82. CrossRef Medline

Wesson DW, Donahou TN, Johnson MO, Wachowiak M (2008b) Sniffing behavior of mice during performance in odor-guided tasks. Chem Senses 33:581-596. CrossRef Medline

Wesson DW, Verhagen JV, Wachowiak M (2009) Why sniff fast? The relationship between sniff frequency, odor discrimination, and receptor neuron activation in the rat. J Neurophysiol 101:1089-1102. Medline

Yang GC, Scherer PW, Mozell MM (2007a) Modeling inspiratory and expiratory steady-state velocity fields in the Sprague-Dawley rat nasal cavity. Chem Senses 32:215-223. Medline 
Yang GC, Scherer PW, Zhao K, Mozell MM (2007b) Numerical modeling of odorant uptake in the rat nasal cavity. Chem Senses 32:273-284. Medline Youngentob SL, Mozell MM, Sheehe PR, Hornung DE (1987) A quantita- tive analysis of sniffing strategies in rats performing odor detection tasks. Physiol Behav 41:59-69. CrossRef Medline 